\title{
Multiobjective Optimization Design and Experimental Investigation on the Axial Flow Pump with Orthogonal Test Approach
}

\author{
Yuquan Zhang $\mathbb{D}^{1},{ }^{1}$ Yanhe Xu ${ }^{D},{ }^{2}$ Yuan Zheng $\mathbb{D D}^{1},{ }^{1}$ E. Fernandez-Rodriguez, ${ }^{3}$ Aoran Sun, ${ }^{4}$ \\ Chunxia Yang $\oplus^{1}$, and Jue Wang $\mathbb{1}^{5}$ \\ ${ }^{1}$ College of Energy and Electrical Engineering, Hohai University, Nanjing 210098, China \\ ${ }^{2}$ School of Hydropower and Information Engineering, Huazhong University of Science and Technology, Wuhan 430074, China \\ ${ }^{3}$ Technological Institute of Merida, Technological Avenue, Merida 97118, Mexico \\ ${ }^{4}$ Anhui Survey and Design Institute of Water Conservancy and Hydropower, Hefei 230088, China \\ ${ }^{5}$ College of Mechanical and Electrical Engineering, Hohai University, Changzhou 213022, China
}

Correspondence should be addressed to Yanhe Xu; yh_xu@hust.edu.cn

Received 11 September 2019; Revised 21 October 2019; Accepted 28 October 2019; Published 5 December 2019

Academic Editor: Sing Kiong Nguang

Copyright ( 2019 Yuquan Zhang et al. This is an open access article distributed under the Creative Commons Attribution License, which permits unrestricted use, distribution, and reproduction in any medium, provided the original work is properly cited.

\begin{abstract}
A multiobjective optimization technique based on the computational fluid dynamics (CFD) simulations and the orthogonal test is proposed to reduce the pressure pulsation in this paper. Three levels of four well-known performance factors $L_{9}\left(3^{4}\right)$ were considered in the orthogonal test scheme: the number of blades, the blade setting angle, the hub ratio, and the distance between the blade and the guide vane. The evaluation indexes corresponded to the head, efficiency, shaft power, and pressure pulsation, respectively. An optimal configuration $\mathrm{A}_{2} \mathrm{~B}_{1} \mathrm{C}_{2} \mathrm{D}_{3}$ was obtained by comprehensive frequency analysis method, after intuitive and range analysis. In comparison with the nonoptimized model, the new design's head and efficiency increased by $17.8 \%$ and $4.26 \%$, whilst the shaft power and the pressure pulsation coefficient reduced by $1.22 \%$ and $11 \%$, respectively. Experiments conducted on the optimized pump were consistent with the CFD model. Six different rotational speed conditions in the optimal operating points were numerically calculated in order to explore the internal hydraulic characteristics of the optimized axial flow pump. It is verified that the comprehensive frequency analysis method based on the orthogonal test approach is effective for the multiobjective optimization of the axial flow pump.
\end{abstract}

\section{Introduction}

Pump is a kind of widely used machinery with universal applications which accounts for almost $22 \%$ of the world's energy consumed by electric motors [1-4]. Axial flow pumps, as the main pump type applied in low-lift and large-flow pumping stations, have attracted increasing attention in field irrigation and water diversion projects [5-7]. The internal flow of an axial flow pump is a three-dimensional complex flow field producing pressure pulsations and thus affecting the stability of the device $[8,9]$. Therefore, it is of great significance to improve the pump efficiency and stability in order to meet targets of worldwide energy saving and security operation.
Although several approaches exist for the purpose of multiobjective optimization design of the pumps and impellers, programs using computational fluid dynamics (CFD) continues to be the most popular method [10-12]. These programs, when run by high-performance computers, can forecast not only the functioning of the pump but also the flow field upstream and downstream of the pump [13-15]. For instance, Zhang et al. [16] validated a CFD model, used for optimizing the submerged propeller operated in an oxidation ditch. The results showed that the CFD model is able to characterize the flow pattern and energy consumption in the full-scale oxidation ditch. In a similar way, Shojaeefard et al. [17] simulated the 3D flow in the 
pump along with the volute by the CFD method, under different cases of passage width and outlet angle of the impeller. The pump performance was shown to improve with the modification of the original geometry because of the reduction of losses in the passage and outlet of the impeller. Asuaje et al. [18] conducted a 3D flow simulation by CFD tools based on the impeller performance. Spence and Amaral-Teixeira [19] presented a CFD approach for the analysis of the geometrical variations on the performance characteristics of a pump. Geometric recommendations were put forward to reducing vibration and increasing component life through the reductions in pressure pulsations. Fleder and Böhle [20] investigated the effect of blade number on the flow characteristics and performance of different pumps by CFD simulations and experiments. It is shown that the number of blades has a significant influence on the circulation velocity and flow angles between blades and side channel. Yang et al. [21] numerically researched the effect of adjustable guide vane on the pump performance with seven different adjustable angles. The results indicated that the hydraulic efficiency of the pump could be improved considerably by adjusting the guide vane angle so as to decrease the flow separation. Hou et al. [22] selected the geometrical parameter of blade setting angle to design a centrifugal pump. It was found that the optimal pump has an improved efficiency. A study on the axial flow pump performance improvement was presented by Park et al. [23], where the effect of hub tip ratio was investigated to design the guide vanes, and the performance of the pump was enhanced compared with the original model. Liu et al. [24] studied the centrifugal pump performance by extending the axial distances from $280 \mathrm{~mm}$ to $460 \mathrm{~mm}$ using experiments and CFD simulation. The results showed that the axial distance has a remarkable impact on the pressure fluctuations. Bonaiuti et al. [25] described the parametric design of a mixed-flow pump. CFD analyses were applied to assess the hydrodynamic performance of the different impeller and diffuser geometry configurations. A good agreement was found between experimental results and numerical simulations. It is indicated that the parametric study could provide a design guideline so as to find the optimal compromise in the process of pump design.

The orthogonal test is a useful and statistical method using the representative parameter configuration to achieve a fast optimization process [26-29]. It was developed by Byrne [30] and plays an important role in the design of efficient pumps. Xu et al. [31] introduced an orthogonal method for designing a high-efficient, low-cost centrifugal pump. An orthogonal table designation L16 was established, where 16 individuals of blades were generated using 5 parameters. A centrifugal pump was suggested, having a better efficiency and cavitation performance than the original one. In Zhou et al.'s study [32], a pump was designed using 16 impellers and 5 main impeller geometric parameters; the pump efficiency and head of the optimal pump had a significant improvement compared with the original pump. Wang et al. [33] established a function relationship between three factors of impeller and the efficiency through the quadratic regression orthogonal test. The three factors included the impeller blade outlet width, the impeller blade outlet stagger angle, and the impeller outlet slope. The results demonstrated that orthogonal test is an effective method to improve the efficiency of stainless steel stamping multistage pump. Shi et al. [34] designed 16 schemes concerning the different flow conditions of the passages with orthogonal test to investigate the hydraulic performance of an axial flow pump device. The results showed that an optimized scheme of the inlet and outlet passages could be obtained by the approach of orthogonal test.

It can be seen from the above literature review that CFD is a reliable and convenient approach to optimize the pump efficiency, and orthogonal test is a credible and effective method for pump optimization. Currently, lots of efforts have been made by the researchers on the prediction of pump's overall performance, and majority of these studies mainly focused on the pump efficiency and power as the optimization index rather than on the pressure pulsation and stability of the pump. In fact, the pressure pulsation can lead to dangerous consequences with regard to pump reliability which may ultimately lead to device failure. Pressure pulsation has not been considered as the optimization parameter in the method of orthogonal test in previous studies, and no studies have reported the comprehensive frequency analysis method for the axial flow pump optimization. Therefore, it is paramount to improve the stability of the axial flow pump considering the flow rate, head, and shaft power to meet the design requirements.

In the present paper, we propose a geometrical optimization technique to reduce the pressure pulsation and improve the overall performance of an axial flow pump, based on the CFD method with the implementation of the orthogonal test approach. Four factors influencing pump functioning were taken into account, namely, the number of blades, the blade setting angle, the hub ratio, and the distance between blade and guide vane. Parameters were analyzed in terms of the flow rate, head, efficiency, shaft power, and pressure pulsation. Finally, four design parameters, along with an optimal pump, were determined by means of the comprehensive frequency analysis method. Experiments were conducted to verify the method, and six different rotational speed conditions in the optimal operating points were numerically calculated.

\section{Orthogonal Test Theory}

2.1. Method of the Orthogonal Test. The traditional optimization method is mainly used to transform a multi-index into a single-index model analysis. The purpose of the method is to test the influence of a particular factor over the whole outcome, with the view of obtaining optimal configuration in terms of the performance levels [35]. However, enormous workload occurs, when the comprehensive test consists of many horizontal combinations. Overall, the traditional comprehensive test method is considered laborious and inefficient [36]. By contrast, the orthogonal test method uses an orthogonal table to analyze and arrange the multifactor test [37]. In this method, only some representative factors from the whole level combinations are selected, 
referred to as a partial test. This procedure allows a formal solution to be found, thus requiring less workload than the comprehensive test method.

The basic procedure of the orthogonal test is indicated in Figure 1. It is made up of the design of the scheme and the analysis of the test results.

In this paper, the influence and weight of four factors were taken to analyze the pump performance. These include the number of blades (factor A), blade setting angle (factor B), hub ratio (factor C), and distance between blade and guide vane (factor $D$ ). The number of test schemes was 9 , and the level of combination per factor was 3 , and thus $L_{n}\left(a^{\mathrm{b}}\right)=$ $L_{9}\left(3^{4}\right)$. Table 1 details the parameters of the axial flow pump.

A range analysis method was adopted to clarify the significance of the factors on the pump performance. The factor $k_{i}$ is the sum of the test indexes corresponding to $i$ levers under a certain factor, and its average score is named as $K_{i}$, whilst $R_{\text {ang }}$ is the range between each factor and reflects the difference between the maximal and minimal value of each factor's level. The greater the value of $R_{\mathrm{ang}}$, the greater the effect on the test index [34].

2.2. Determination of the Orthogonal Test Scheme. The numerical setup of Table 2 bears a close resemblance to Table 1 , by using $L_{9}\left(3^{4}\right)$. The pump has a radial variation of blade angle $(\Phi)$ as depicted in Figure 2. The relative radius $(r / R)$ is defined as the radius of the section $(r)$ to the blade radius $(R)$.

\section{Numerical Simulation}

3.1. Simulation Methodology. The flows for the axial flow pump are highly turbulent, and the most common tool to model them is through the solution of the Reynolds-averaged Navier-Stokes (RANS) equations [1]. The given approach saves computational resource significantly, compared with other methods such as large eddy simulations [38]. RANS equations use the Reynolds decomposition of velocity and pressure as follows:

$$
\begin{gathered}
\text { continuity }: \frac{\partial \overline{u_{i}}}{\partial x_{i}}=0 \\
\text { momentum }: \rho \frac{\partial \overline{u_{i}}}{\partial t}+\rho \frac{\partial\left(\overline{u_{i} u_{j}}\right)}{\partial x_{j}}=\rho \bar{f}_{i}+\frac{\partial}{\partial x_{j}}\left(-\bar{p} \delta_{i j}+2 \mu \bar{S}_{i j}-\rho \overline{u_{i}^{\prime} u_{j}^{\prime}}\right)
\end{gathered}
$$

where $u_{i}$ is velocity, $x_{i}$ is the spatial coordinate, $p$ is the pressure, $\rho$ is the fluid density, $\mu$ is the dynamic viscosity, $\bar{f}_{i}$ is the time mean external force tensor, and $\bar{S}_{i j}=0.5\left(\partial \overline{u_{i}} / \partial x_{j}+\partial \overline{u_{j}} / \partial x_{i}\right)$ is the mean rate of the strain tensor. The Dirac function $\delta_{i j}=1$ for $i=j$ and equals zero otherwise. The Reynolds stresses $\overline{u_{i}^{\prime} u_{j}^{\prime}}$ are solved by using a turbulence model to closure the RANS equations. The $k-\omega$ SST (Shear Stress Transport) model, proposed by Menter [39], combines the best of both standard $k-\omega$ and $k-\varepsilon$ turbulence models. SST model performs better in applications where adverse pressure gradients occur, so it is adopted in this study [40].
3.2. Boundary Condition and Mesh Refinement Study. The original axial flow pump of Jiangdu No. 1 pump station has been operating for many years, and as it is known, the practical operating mode always deviates from the design conditions. The computational domain includes the elbow-type inlet passage section, the blades, the guide vane, the siphon outlet flow section, and the outlet bend, as shown in Figure 3. The basic parameters are as follows: design flow rate $Q=298.6 \mathrm{~L} / \mathrm{s}$, head $H=6.8 \mathrm{~m}$, rotor's rotational speed $n=1461 \mathrm{r} / \mathrm{min}$, and number of blades $Z=4$. An improved design provided a design flow of $Q=328.5 \mathrm{~L} / \mathrm{s}$ and a head of $H=7.8 \mathrm{~m}$.

ICEM CFD software was used to obtain the mesh of the computing domain [14]. The unstructured grids were adopted due to its strength in the adaptability of the complicated and irregular geometry. In the domain of the blades and guide vanes, unstructured mesh with prisms was used to approximate the geometry of runner and guide vane. The $y^{+}$, the dimensionless wall distance of the grid cells adjacent to the pump surface, was within 30 to 200 , a range sufficiently enough for capturing turbulent flow near the surfaces.

Ansys CFX was used to simulate the whole flow passage of the nine test schemes [16]. In order to transfer the data between the interfaces, the dynamic-static interface was set as Frozen Rotor Interface in the steady calculation and as Transient-Frozen Rotor/Stator interface in the unsteady calculation. The loss caused by small gaps between the pump body and the impeller was ignored. Mass flow and free outlet boundary condition were used to describe the inlet and outlet flow, respectively.

For the unsteady simulation, the blades rotated three degrees at each time step. Periodic behavior was obtained by eight complete rotations. Four sets of pressure pulsation monitoring points were set up around the blades and guide vanes to obtain the pressure pulsation information inside the axial flow pump as shown in Figure 4. The results of the last two cycles in the simulation were taken as the data for pressure pulsation analysis. Pressure pulsation coefficient $\left(C_{\mathrm{P}}\right)$ was introduced to describe the pressure pulsation characteristics:

$$
C_{\mathrm{p}}=\left|\frac{P_{i}-P_{\mathrm{avc}}}{P_{\mathrm{avc}}}\right|,
$$

where $C_{\mathrm{p}}$ is the pressure pulsation coefficient, $P_{i}$ refers to the pressure at the monitoring point, and $P_{\text {avc }}$ is the averaged static pressure within the rotation period.

Table 3 shows the three mesh densities with coarse, medium, and fine levels whilst Table 4 illustrates the variation of the evaluation indexes. It is known that cases with a higher mesh density usually obtain better simulation results. However, the difference between fine and medium meshes is less than $2 \%$ which is negligible. Therefore, medium meshes were used considering both the computation accuracy and required resources.

\section{Results and Discussion}

The results of the nine test schemes simulated by CFD with the designed flow rate are shown in Table 5. 


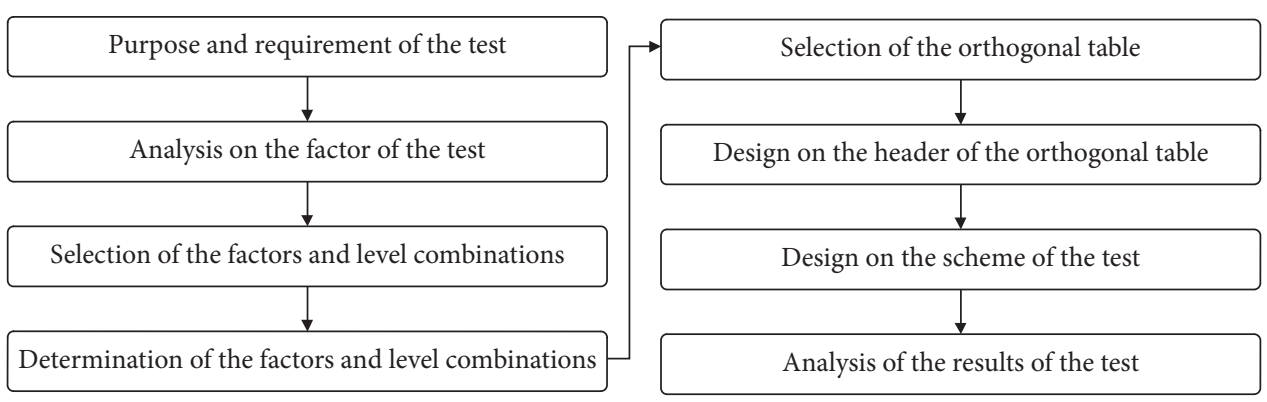

FIGURE 1: Basic design procedure of the orthogonal test.

TABle 1: Orthogonal table $L_{9}\left(3^{4}\right)$.

\begin{tabular}{|c|c|c|c|c|c|}
\hline \multirow{2}{*}{ Test scheme } & \multicolumn{4}{|c|}{ Test factor } & \multirow{2}{*}{ Factors and level combinations } \\
\hline & A & $\mathrm{B}$ & $\mathrm{C}$ & $\mathrm{D}$ & \\
\hline 1 & 1 & 1 & 1 & 1 & $\mathrm{~A}_{1} \mathrm{~B}_{1} \mathrm{C}_{1} \mathrm{D}_{1}$ \\
\hline 2 & 1 & 2 & 2 & 2 & $\mathrm{~A}_{1} \mathrm{~B}_{2} \mathrm{C}_{2} \mathrm{D}_{2}$ \\
\hline 3 & 1 & 3 & 3 & 3 & $\mathrm{~A}_{1} \mathrm{~B}_{3} \mathrm{C}_{3} \mathrm{D}_{3}$ \\
\hline 4 & 2 & 1 & 2 & 3 & $\mathrm{~A}_{2} \mathrm{~B}_{1} \mathrm{C}_{2} \mathrm{D}_{3}$ \\
\hline 5 & 2 & 2 & 3 & 1 & $\mathrm{~A}_{2} \mathrm{~B}_{2} \mathrm{C}_{3} \mathrm{D}_{1}$ \\
\hline 6 & 2 & 3 & 1 & 2 & $\mathrm{~A}_{2} \mathrm{~B}_{3} \mathrm{C}_{1} \mathrm{D}_{2}$ \\
\hline 7 & 3 & 1 & 3 & 2 & $\mathrm{~A}_{3} \mathrm{~B}_{1} \mathrm{C}_{3} \mathrm{D}_{2}$ \\
\hline 8 & 3 & 2 & 1 & 3 & $\mathrm{~A}_{3} \mathrm{~B}_{2} \mathrm{C}_{1} \mathrm{D}_{3}$ \\
\hline 9 & 3 & 3 & 2 & 1 & $\mathrm{~A}_{3} \mathrm{~B}_{3} \mathrm{C}_{2} \mathrm{D}_{1}$ \\
\hline
\end{tabular}

TABLE 2: Orthogonal table of the axial flow pump.

\begin{tabular}{|c|c|c|c|c|}
\hline \multirow{2}{*}{$\begin{array}{l}\text { Orthogonal test } \\
\text { scheme }\end{array}$} & \multicolumn{4}{|c|}{ Four test factors } \\
\hline & Number of blades (A) & Blade setting angle (B) & Hub ratio $(\mathrm{C})$ & Distance between blade and guide vane (D) $(\mathrm{mm})$ \\
\hline 1 & 3 & $\mathrm{~B}_{1}$ & 0.45 & 175 \\
\hline 2 & 3 & $\mathrm{~B}_{2}$ & 0.47 & 210 \\
\hline 3 & 3 & $\mathrm{~B}_{3}$ & 0.49 & 263 \\
\hline 4 & 4 & $\mathrm{~B}_{1}$ & 0.47 & 263 \\
\hline 5 & 4 & $\mathrm{~B}_{2}$ & 0.49 & 175 \\
\hline 6 & 4 & $\mathrm{~B}_{3}$ & 0.45 & 210 \\
\hline 7 & 5 & $\mathrm{~B}_{1}$ & 0.49 & 210 \\
\hline 8 & 5 & $\mathrm{~B}_{2}$ & 0.45 & 263 \\
\hline 9 & 5 & $\mathrm{~B}_{3}$ & 0.47 & 175 \\
\hline
\end{tabular}

Each data was analyzed by the intuitively single-index method, followed by the range analysis. Finally, the comprehensive frequency analysis method was used to obtain the optimized levels.

4.1. Intuitive and Range Analysis. Flow rate is a working parameter belonging to the specified index which is different from the evaluation indexes of efficiency and pressure pulsation. It is our goal to increase the efficiency and reduce the pressure pulsation. The head belongs to the criterion index, and thus, only the schemes with head meeting the criterion could be available in the optimization process. Based on the results shown in Table 5, the head of No. 1 , No. 2, No. 3 , No. 5, No. 6, and No. 7 test schemes did not meet the requirement at the condition of the designed flow rate, and thus, they were identified as nonoptimal models.
The optimal test scheme, considering the index of head, was No. $4\left(\mathrm{~A}_{2} \mathrm{~B}_{1} \mathrm{C}_{2} \mathrm{D}_{3}\right)$, compared with the index of efficiency's No. $9\left(\mathrm{~A}_{3} \mathrm{~B}_{3} \mathrm{C}_{2} \mathrm{D}_{1}\right)$, shaft power's No. $8\left(\mathrm{~A}_{3} \mathrm{~B}_{2} \mathrm{C}_{1} \mathrm{D}_{3}\right)$, and pressure pulsation coefficient's No. $4\left(\mathrm{~A}_{2} \mathrm{~B}_{1} \mathrm{C}_{2} \mathrm{D}_{3}\right)$.

As mentioned in the above part of orthogonal test theory, range analysis is applied to clarify the significance levels of four influencing factors on the evaluation indexes. $k_{i}$ is calculated by the total of the test indices corresponding to $i$ levers whilst $K_{i}$ indicates the average value. $R_{\text {ang }}$ is subtracted by the maximal and minimal value of $K_{i}$ (Tables 6 and 7).

To verify the degree of factor influence on the four evaluation indexes, a visual comparison was made between the factor level and evaluation indexes. Such cases are depicted in Figures 5(a) 5(d).

As observed, an increase of blade numbers leads to higher head, and the efficiency reaches a peak at $C_{2}$. As the shaft power is in direct proportion to the flow rate and head, the shaft power grows with increased blade numbers, as 


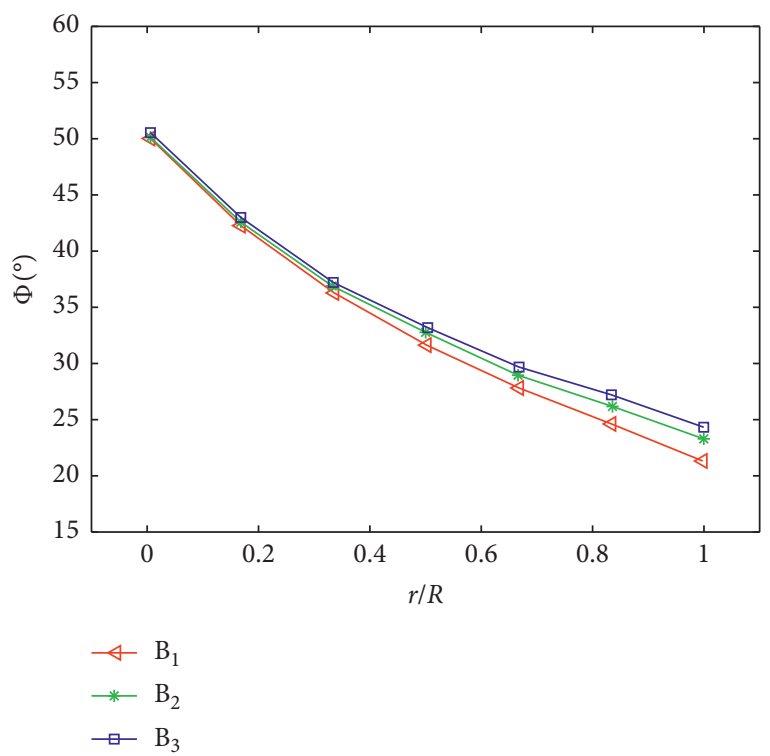

Figure 2: Blade seating angle of each section.

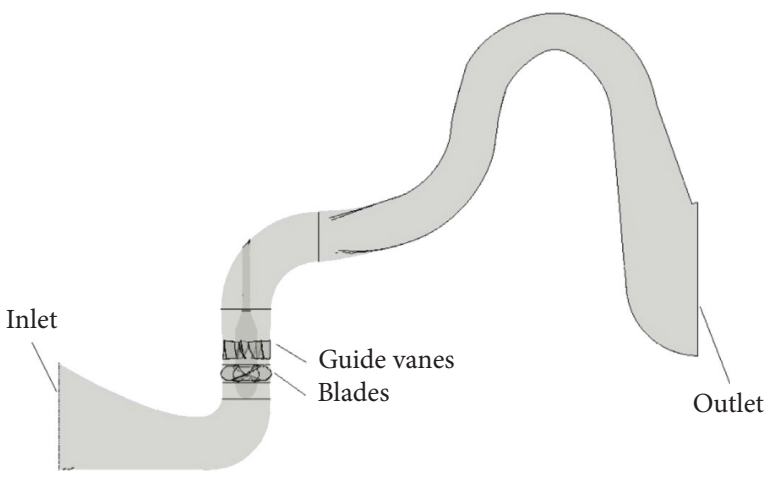

FIgURE 3: Computational domain of the pump.

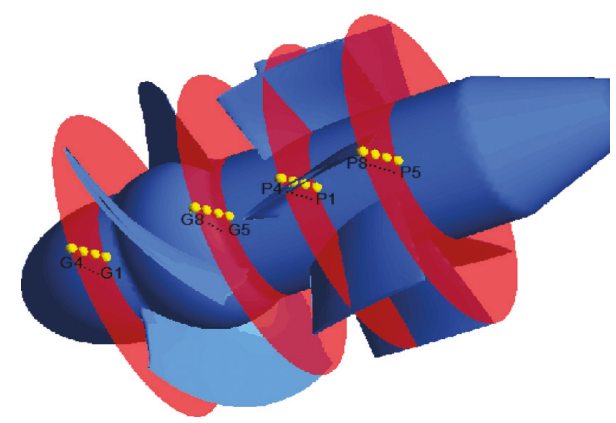

FIGURE 4: Four sets of pressure fluctuation monitoring points.

indicated in Figure 5(c). The number of blades (factor A) has a significant effect on the cascade solidity but has a little effect on the other structures of the pump. As a result, the load on each blade will be reduced with more blades, thus improving the flow pattern around the runner and thus increasing the shaft power [41].

The circulation of velocity $\Gamma$ is calculated by the formula: $\Gamma=2 \pi r V_{\mathrm{u}}$, where $V_{\mathrm{u}}$ is the circular velocity. In order to obtain the same head for each section of the blade, the circulation of velocity of the blade has to be the same in different radial direction, and the radius $r$, inversed with the circular velocity $V_{\mathrm{u}}$. Therefore, the blade setting angle (factor B) near the hub is larger than that of the outer edge of the blade, distorting seriously the blade. Based on Figures $5(a)-5(d)$, a factor level changes from $B_{3}$ to $B_{1}$, resulting in a significant increase of the head and shaft power and slight reduction of the efficiency $(2 \%)$. By reducing the blade seating angle of each section, the distortion degree of blade lowers, improving the power output.

If the factor level of the hub ratio (factor $\mathrm{C}$ ) changes from 0.45 to 0.49 , the head, efficiency, and shaft power increase and decrease to low levels, peaking at $C_{2}$. The smaller the hub ratio, the lesser the hydraulic friction losses and the greater the flow around the pump. However, the product will increase the distortion of the blades, disorder the flow pattern, and lower the efficiency of the pump. The hub ratio has an important influence on the flow pattern around the blades, and thus, it is an important geometric parameter in the hydraulic design and optimization of the axial flow pump.

The distance between the blade and guide vane (factor $\mathrm{D}$ ) was determined according to the formula $S=(0.1 \sim 0.15) D_{\mathrm{h}}$, where $D_{\mathrm{h}}$ is the diameter of the blades [17]. As we can see from Figures $5(a)-5(d)$, factor $D$ has lesser influence on the evaluation indexes, when compared with factors A, B, and C. With the increase of the distance between the blade and guide vane, the head and shaft power increase monotonously and the efficiency increases but then decreases at higher levels.

Through the above range analysis, the optimized test schemes can be obtained as follows: test scheme with optimum index of head was $A_{3} B_{1} C_{2} D_{3}$, of efficiency was $A_{3} B_{3} C_{2} D_{2}$, of shaft power was $A_{1} B_{3} C_{3} D_{1}$, and of pressure pulsation was $\mathrm{A}_{2} \mathrm{~B}_{1} \mathrm{C}_{2} \mathrm{D}_{1}$. On the other hand, from the calculated result of range $R$, the impact from largest to smallest on head was $\mathrm{A}>\mathrm{C}>\mathrm{B}>\mathrm{D}$, as opposed to efficiency's $\mathrm{C}>\mathrm{B}>\mathrm{A}>\mathrm{D}$, shaft power's $\mathrm{A}>\mathrm{C}>\mathrm{B}>\mathrm{D}$, and pressure pulsation's $\mathrm{A}>\mathrm{B}>\mathrm{C}>\mathrm{D}$.

4.2. Comprehensive Frequency Analysis. The four evaluation indexes are of equal importance. Table 8 summarizes the results of the three levels corresponding to four factors, based on the test schemes obtained by the intuitive analysis and the test schemes obtained by the range analysis.

Table 8 shows $A_{2}$ and $A_{3}$ occur with the highest frequency $(3 / 8)$. For factor $B$, the values are $B_{1}$ and $B_{3}(1 / 2)$ and for factor $C, C_{2}(3 / 4)$, whilst for factor $D, D_{3}(1 / 2)$. Consequently, the preliminary optimum test scheme becomes $\mathrm{A}_{2} / \mathrm{A}_{3} \mathrm{~B}_{1} / \mathrm{B}_{3} \mathrm{C}_{2} \mathrm{D}_{3}$.

The pump's specific speed is estimated by equation (4), where $n_{\mathrm{s}}$ is the specific speed of the pump. Since from Section 4.1, an increase of the number of blades corresponded to both lower specific speeds and the anticavitation performance of the pump. Therefore, a factor level of $\mathrm{A}_{2}$ with low number of blades becomes suitable for the model:

$$
n_{\mathrm{s}}=\frac{3.65 n \sqrt{Q}}{H^{3 / 4}} \text {. }
$$


TABLE 3: Mesh size for the calculation model (million).

\begin{tabular}{lcccc}
\hline Mesh density & Number of elements (million) & Head $(\mathrm{m})$ & Shaft power $(\mathrm{kW})$ & Efficiency \\
\hline Coarse & 1.51 & 6.23 & 15.67 & 68.52 \\
Medium & 3.39 & 6.42 & 14.92 & 72.12 \\
Fine & 4.26 & 6.50 & 14.82 & 72.26 \\
\hline
\end{tabular}

TABLE 4: Variation of the evaluation indexes.

\begin{tabular}{|c|c|c|c|c|}
\hline Mesh density & Number of elements (million) & Head $(m)$ & Shaft power $(\mathrm{kW})$ & Efficiency \\
\hline Coarse & 1.51 & $4.2 \%$ & $5.7 \%$ & $5.2 \%$ \\
\hline Medium & 3.39 & $1.2 \%$ & $0.7 \%$ & $0.2 \%$ \\
\hline Fine & 4.26 & - & - & - \\
\hline
\end{tabular}

TABLE 5: Results of the nine test schemes.

\begin{tabular}{lcccc}
\hline Test schemes & & & Evaluation indexes \\
Efficiency, $\eta(\%)$ & Pressure pulsation coefficient, $C_{\mathrm{p}}$ \\
\hline 1 & Head, $H(\mathrm{~m})$ & Shaft power, $P(\mathrm{~kW})$ & 72.12 & 1.19 \\
2 & 6.42 & 14.92 & 78.47 & 1.16 \\
3 & 6.58 & 16.64 & 72.66 & 1.11 \\
4 & 5.59 & 20.29 & 78.59 & 0.31 \\
5 & 8.01 & 15.67 & 72.56 & 0.80 \\
6 & 6.70 & 16.74 & 75.28 & 0.74 \\
7 & 6.90 & 17.58 & 72.94 & 0.53 \\
8 & 7.48 & 18.47 & 74.25 & 0.99 \\
9 & 7.72 & 19.76 & 79.65 & 0.34 \\
\hline
\end{tabular}

TABLE 6: Range analysis of the results on head and efficiency.

\begin{tabular}{lcccccccc}
\hline Range & \multicolumn{4}{c}{ Head, $H(\mathrm{~m})$} & \multicolumn{4}{c}{ Shaft power, $P(\mathrm{~kW})$} \\
analysis & $\mathrm{A}$ & $\mathrm{B}$ & $\mathrm{C}$ & $\mathrm{D}$ & $\mathrm{A}$ & $\mathrm{B}$ & $\mathrm{C}$ & $\mathrm{D}$ \\
\hline$k_{1}$ & 18.59 & 21.91 & 21.04 & 20.82 & 44.65 & 52.79 & 50.13 & 50.35 \\
$k_{2}$ & 21.61 & 21.00 & 22.29 & 20.96 & 52.70 & 50.78 & 56.69 & 50.96 \\
$k_{3}$ & 22.90 & 20.19 & 19.77 & 21.32 & 55.81 & 49.59 & 46.34 & 51.85 \\
$K_{1}$ & 6.20 & 7.30 & 7.01 & 6.94 & 14.88 & 17.60 & 16.71 & 16.78 \\
$K_{2}$ & 7.20 & 7.00 & 7.43 & 6.99 & 17.57 & 16.93 & 18.90 & 16.99 \\
$K_{3}$ & 7.63 & 6.73 & 6.59 & 7.11 & 18.60 & 16.53 & 15.45 & 17.28 \\
$R_{\text {ang }}$ & 1.43 & 0.57 & 0.84 & 0.17 & 3.72 & 1.07 & 3.45 & 0.50 \\
\hline
\end{tabular}

TABLE 7: Range analysis of the results on shaft power and pressure pulsation coefficient.

\begin{tabular}{lcccccccc}
\hline \multirow{2}{*}{$\begin{array}{l}\text { Range } \\
\text { analysis }\end{array}$} & \multicolumn{3}{c}{ Efficiency, $\eta(\%)$} & \multicolumn{4}{c}{$\begin{array}{c}\text { Pressure pulsation } \\
\text { coefficient, } C_{\mathrm{p}}\end{array}$} \\
& $\mathrm{A}$ & $\mathrm{B}$ & $\mathrm{C}$ & $\mathrm{D}$ & $\mathrm{A}$ & $\mathrm{B}$ & $\mathrm{C}$ & $\mathrm{D}$ \\
\hline$k_{1}$ & 223.25 & 223.65 & 221.65 & 224.33 & 3.46 & 2.03 & 2.92 & 2.33 \\
$k_{2}$ & 226.43 & 225.28 & 236.71 & 226.69 & 1.85 & 2.95 & 1.81 & 2.43 \\
$k_{3}$ & 226.84 & 227.59 & 218.16 & 225.50 & 1.86 & 2.19 & 2.44 & 2.41 \\
$K_{1}$ & 74.42 & 74.55 & 73.88 & 74.78 & 1.15 & 1.02 & 0.97 & 0.78 \\
$K_{2}$ & 75.48 & 75.09 & 78.90 & 75.56 & 0.616 & 1.48 & 0.60 & 0.81 \\
$K_{3}$ & 75.61 & 75.86 & 72.72 & 75.17 & 0.62 & 1.10 & 0.81 & 0.80 \\
$R_{\text {ang }}$ & 1.19 & 1.31 & 6.18 & 0.78 & 0.534 & 0.46 & 0.37 & 0.03 \\
\hline
\end{tabular}

Similarly, a factor lever of $\mathrm{B}_{1}$ with a smaller blade seating angle was adopted, on the basis of the serious distortion applied to the blades and the higher requirements for material and production technology of the blades.
From the above comprehensive frequency analysis, the optimum test scheme was determined as $\mathrm{A}_{2} \mathrm{~B}_{1} \mathrm{C}_{2} \mathrm{D}_{3}$, using a four-bladed pump with a blade seating angle of $B_{1}$, hub ratio of 0.47 , and distance between the blade and guide vane of $263 \mathrm{~mm}$.

4.3. Performance of the Optimized Pump. The optimized test scheme $\mathrm{A}_{2} \mathrm{~B}_{1} \mathrm{C}_{2} \mathrm{D}_{3}$, corresponding to scheme 4 under the design flow of $Q=328.5 \mathrm{~L} / \mathrm{s}$, was further verified in this section.

The first main difference between the two models concerns with the streamlines around the blades and guide vanes at the half of the blade height. As observed in Figure 6, the flow is more unevenly distributed in the original than in the optimized pump, leading to large hydraulic loss. Furthermore, two largescale vortices are formed on the back of the original's guide vane, compared with almost none in the optimized pump. The second difference relates to the high pressure observed near the hub of the original's blade; this almost disappears on the optimized blade surface as shown in Figure 7.

With regard to the pressure pulsation, $C_{\mathrm{p}}$ increases gradually from hub to the blade tip with a cyclical trend, and the maximum coefficient is three times of the hub's as indicated in Figure 8(a). From Figure 8(b), the flow at the outlet of the blades is affected by the rotor-stator interaction of the blades and guide vanes. The peak frequency of the pressure is seven times larger than the peak frequency of the guide vanes, and this is consistent with the number of the guide vanes. As expected, the amplitude of pressure pulsation increases from the hub to the tip, reaching a relatively higher value at a frequency of four times the rotational's. 


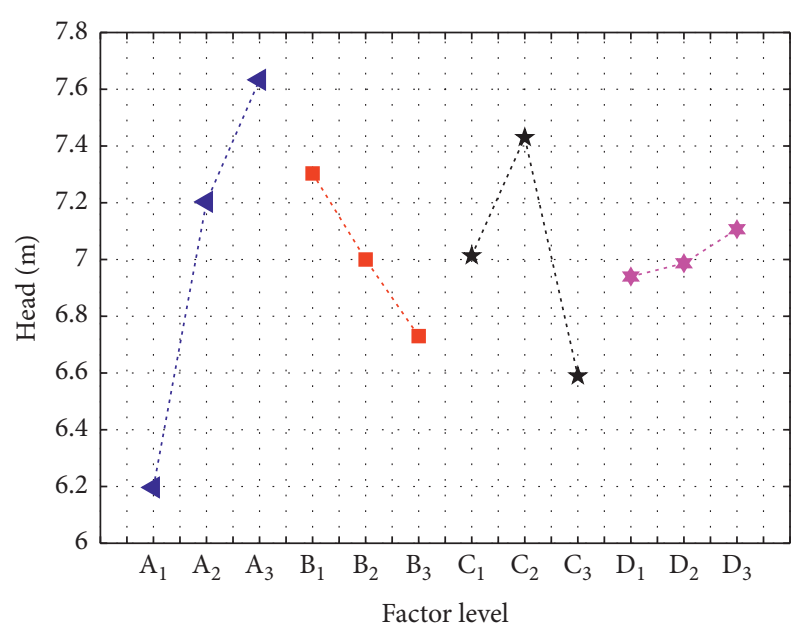

(a)

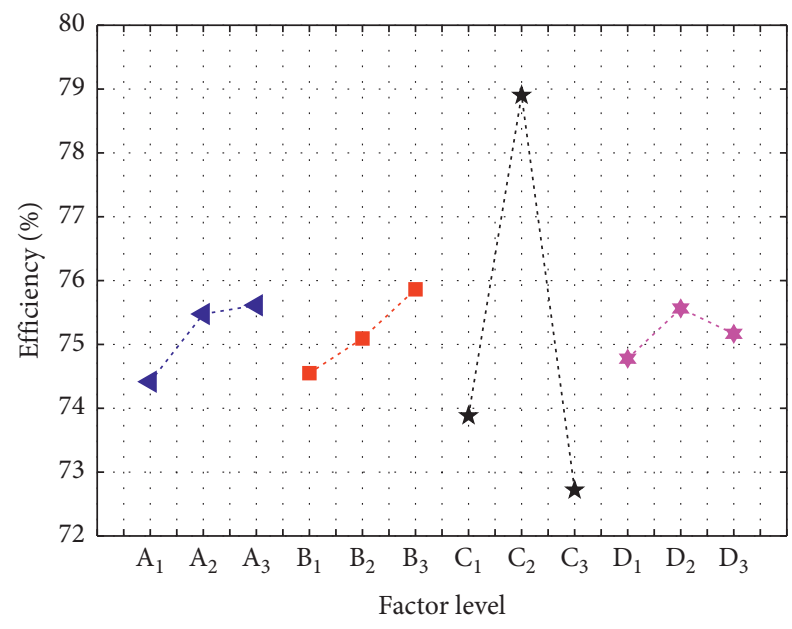

(c)

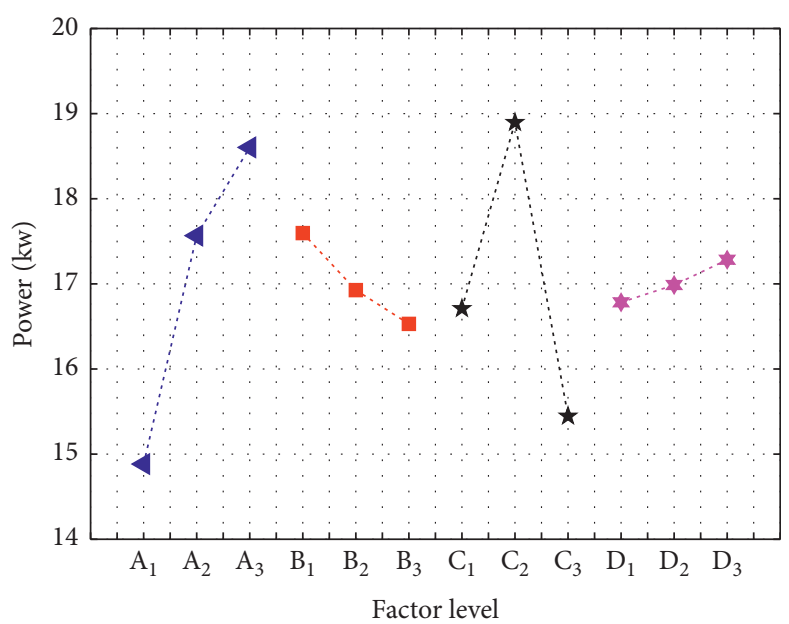

(b)

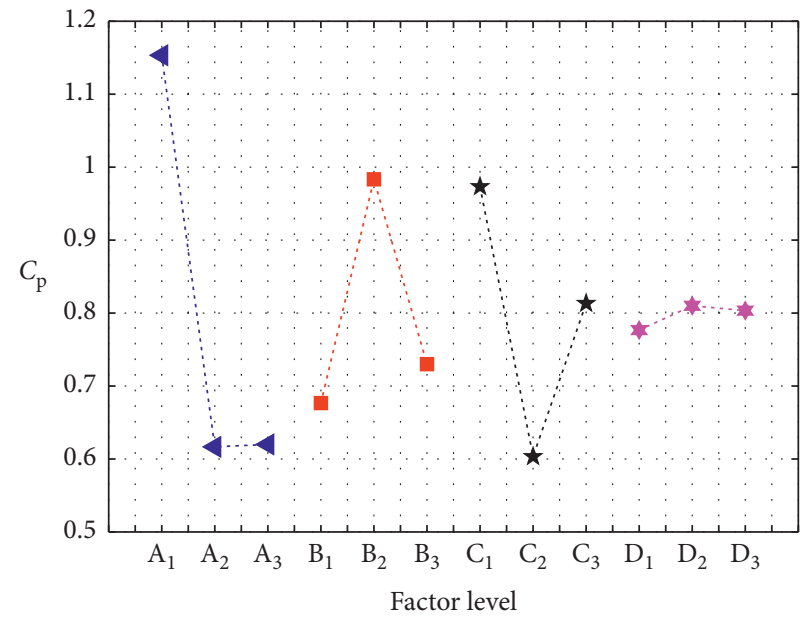

(d)

Figure 5: Relationship between factor lever and four evaluation indexes. (a) Head. (b) Shaft power. (c) Efficiency. (d) Pressure pulsation coefficient.

TABle 8: Comprehensive frequency analysis of three levels corresponding to four factors.

\begin{tabular}{lccccccccccccc}
\hline $\begin{array}{l}\text { Factor } \\
\text { level }\end{array}$ & $\mathrm{A}_{1}$ & $\mathrm{~A}_{2}$ & $\mathrm{~A}_{3}$ & $\mathrm{~B}_{1}$ & $\mathrm{~B}_{2}$ & $\mathrm{~B}_{3}$ & $\mathrm{C}_{1}$ & $\mathrm{C}_{2}$ & $\mathrm{C}_{3}$ & $\mathrm{D}_{1}$ & $\mathrm{D}_{2}$ & $\mathrm{D}_{3}$ \\
\hline Frequency & $1 /$ & $3 /$ & $3 /$ & $1 /$ & 0 & $1 /$ & 0 & $3 /$ & $1 /$ & $3 /$ & $1 /$ & $1 /$ \\
& 4 & 8 & 8 & 2 & 0 & 2 & 0 & 4 & 4 & 8 & 8 & 2 \\
\hline
\end{tabular}

In summary, the optimized axial flow pump is capable of meeting the requirements of the flow rate and head. Under the new design flow rate, the pump efficiency increases from $74.33 \%$ to $78.59 \%$ while the shaft power decreases from $20.54 \mathrm{~kW}$ to $20.29 \mathrm{~kW}$. Besides, the absolute value of the pressure pulsation coefficient reduces by $11 \%$. It is found that the comprehensive frequency analysis is an available method for the multiobjective optimization.

4.4. Comparison between Experiments and Simulation. To verify the method, tests were carried out in the optimized model at the multifunction test bench of Hohai University.
Figure 9 shows the three-dimensional schematic diagram of the hydraulic machinery multifunction test bench. The test bench is a vertical closed circulation system, including tail water tank, pressure water tank, dynamometer motor, and torque meter. Tests were carried out by using the equal head method, where the value of the prototype is the same with the model's. Through the analysis of test error, the total error of this test was $\pm 0.27 \%$, satisfying the standard test regulations.

The physical model of the axial flow pump device is shown in Figure 10. The blades were manufactured with brass material by numerical control machines. To ensure the inner walls of the connecting part are smooth, the inlet and outlet passages were welded with steel plates. The model scale of the pump device is $1: 5.844$.

In order to eliminate the free gases and air bubbles in the system, the pump was operated for more than 30 minutes at rated conditions, prior to the recordings. The pump performance at a certain blade setting angle was investigated under the condition of no cavitation. The measuring points were reasonably distributed on the whole performance 


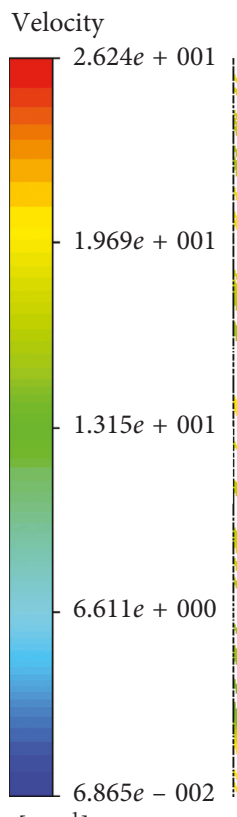

$\left[\mathrm{ms}^{-1}\right]$

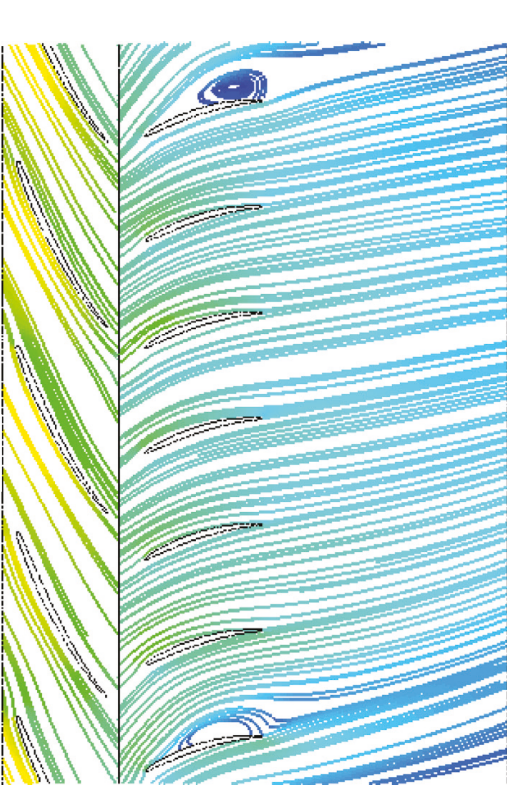

(a)

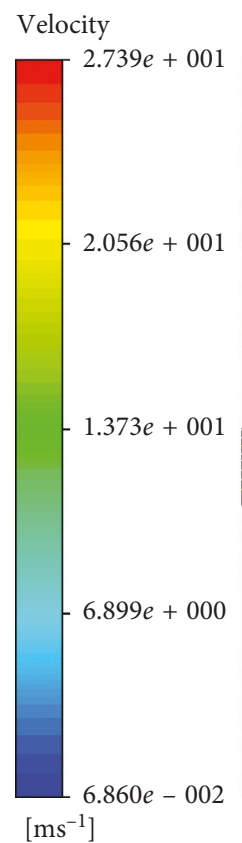

$\left[\mathrm{ms}^{-1}\right]$

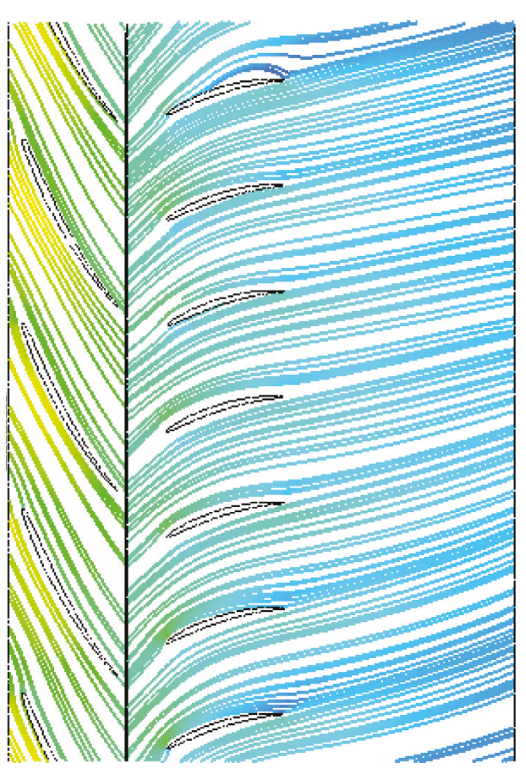

(b)

Figure 6: Distribution of streamline around the blade and guide vane at 0.5 times blade height. (a) Original model. (b) Optimized model.
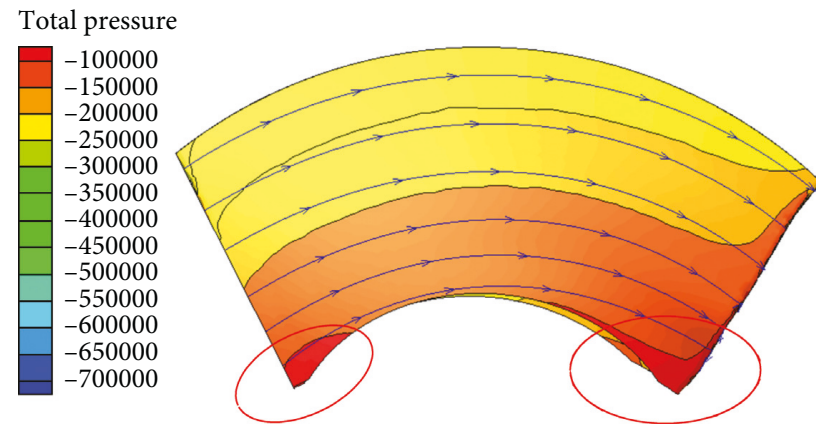

(a)

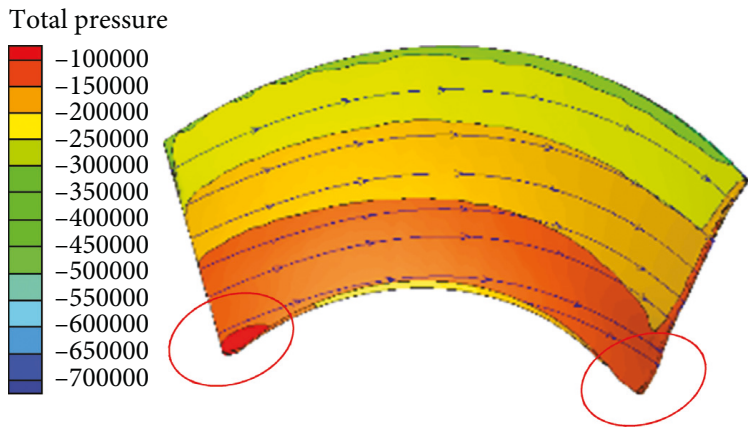

(b)

FiguRE 7: Distribution of pressure contours on the blade surface. (a) Original model. (b) Optimized model.

curve. The data of the flow rate, head, rotate speed, and shaft power were simultaneously obtained and processed, when the parameters remained stable for a period of time.

Figure 11 shows the comparison of the external characteristics between the numerical simulation and the experimental results. The head and the efficiency are slightly higher in the simulations than in the experimental measurements, when the flow rate is smaller than the designed discharge. The maximum error of head and efficiency is $4.6 \%$ and $3.2 \%$, respectively. The small error could be due to the friction losses between the bearings and the seal ring, as well as due to the manual adjustment of the blade's pitch angle. The head and efficiency of the numerical simulation are consistent with the variation of the test results, and the maximum error is not more than 5\%. It shows that the numerical computation model and the method adopted in this paper have a good performance in predicting the external characteristics of the axial flow pump.

A comprehensive characteristic curve of the axial flow pump model was drawn based on the experimental measurements. As shown in Figure 12, the optimized pump model had a wide range of high-efficiency areas. The maximum efficiency of the model device is $78 \%$, with a corresponding head and blade placement angle of $7.52 \mathrm{~m}$ and $0^{\circ}$, respectively. It is obvious that the pump with a blade placement angle of $0^{\circ}$ is able to meet the requirement under the design point.

4.5. Pump Performance under Variable Rotational Speed. Six different rotational speed conditions $(n, 0.9 n, 0.8 n, 0.7 n$, $0.6 n$, and $0.5 n$ ) in the optimal operating points were studied 


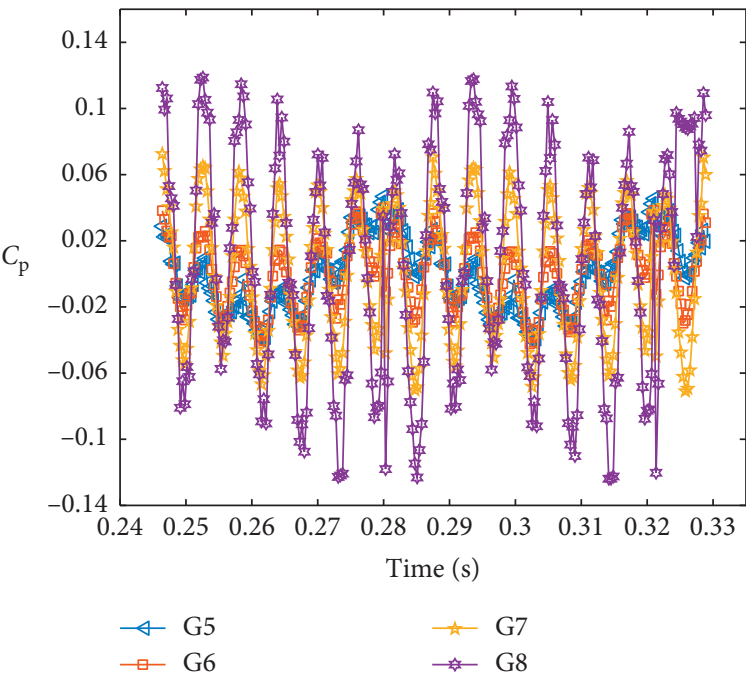

(a)

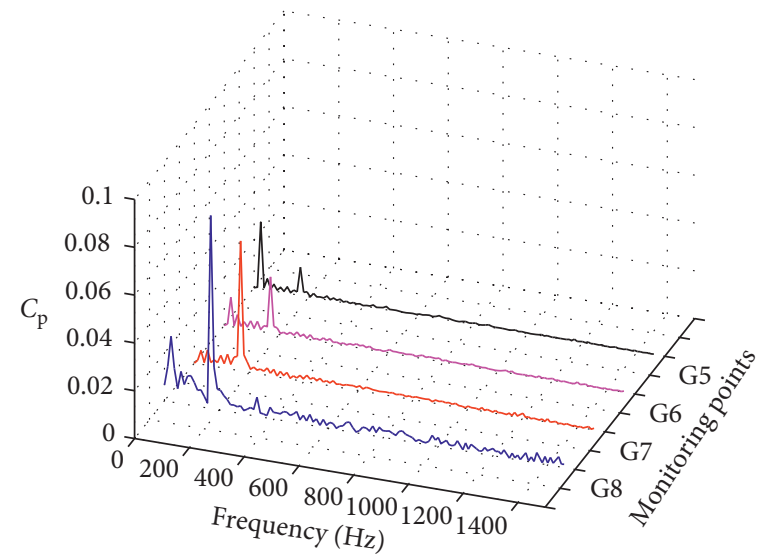

(b)

Figure 8: Pressure pulsation in axial flow pump under design flow rate. (a) Time domain. (b) Frequency domain.

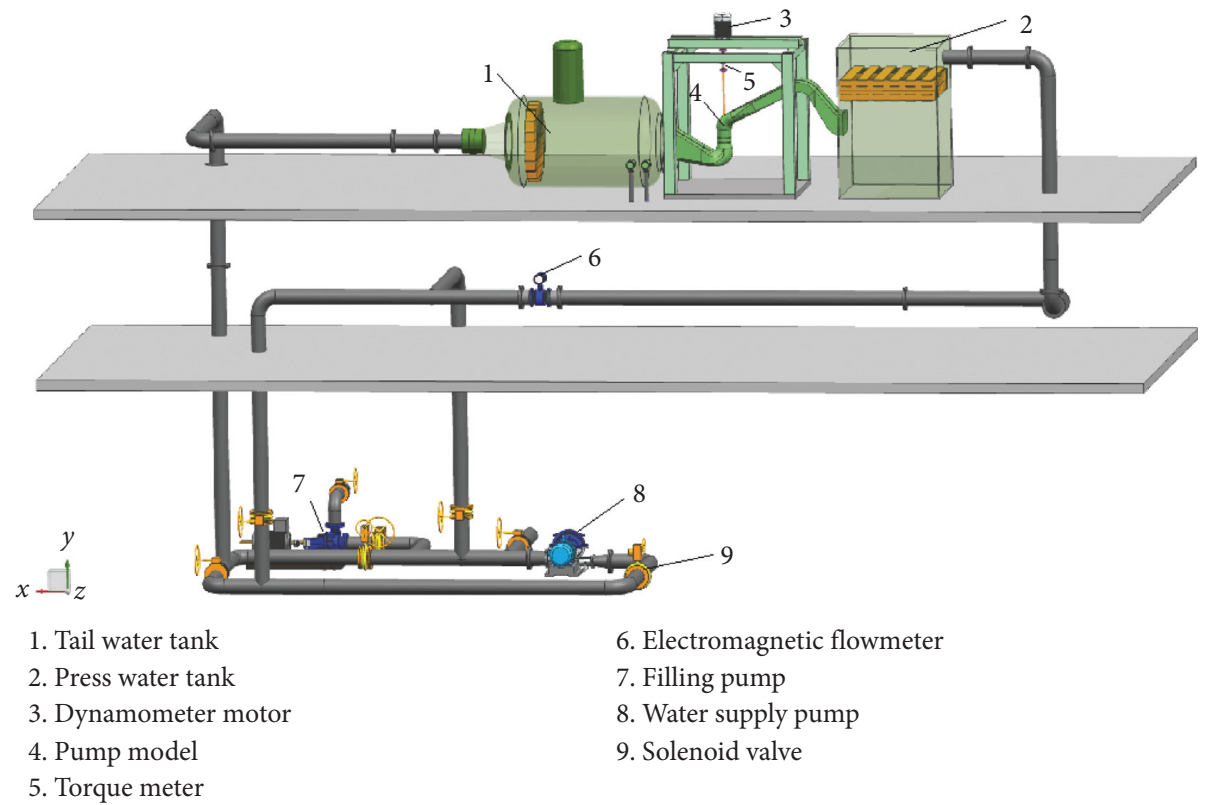

FIgURE 9: Hydraulic multifunction test bench. (1) Tail water tank; (2) press water tank; (3) dynamometer motor; (4) pump model; (5) torque meter; (6) electromagnetic flow meter; (7) filling pump; (8) water supply pump; (9) solenoid valve.

to explore the internal hydraulic characteristics of the optimized axial flow pump.

4.5.1. Hydraulic Performance of the Flow Passage. In an attempt to analyze the influence of rotational speed on the hydraulic loss in the passage of inlet, guide vane, and outlet, the hydraulic loss coefficient $K_{\mathrm{p}}$ is introduced to expound the ratio of hydraulic loss between these three sections and the head of the pump device. The coefficient is defined as follows:

$$
K_{\mathrm{p}}=\frac{\Delta h}{H} \times 100 \% \text {, }
$$

where $\Delta h$ is the hydraulic loss and $\delta$ indicates the ratio of hydraulic loss between different rotational speeds and the rated speed at $1461 \mathrm{r} / \mathrm{min}$. Table 9 shows the hydraulic loss of the passages at variable rotational speed, along with the pump efficiency.

As seen from Table 9, at rated rotational speed, the hydraulic loss ratio of the inlet passage, guide vane passage, and outlet passage is the smallest, whilst the hydraulic efficiency of the blades is the highest at $93.58 \%$. At different rotational speeds, the hydraulic loss ratio $K_{\mathrm{p}}$ increases with the decrease of rotational speed. Evidently, the pump efficiency decreases with lower rotational speeds since speed influences the velocity circulation and the hydraulic loss of 


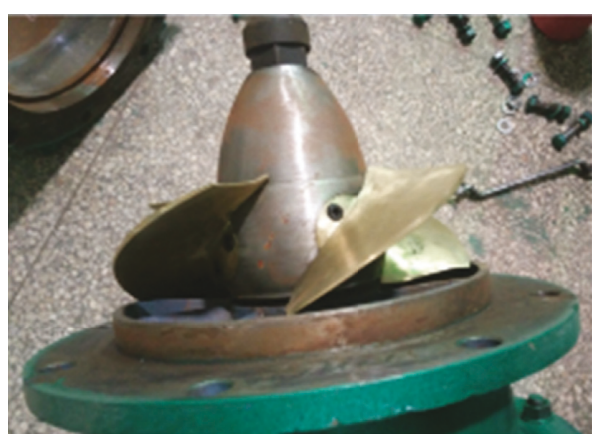

(a)

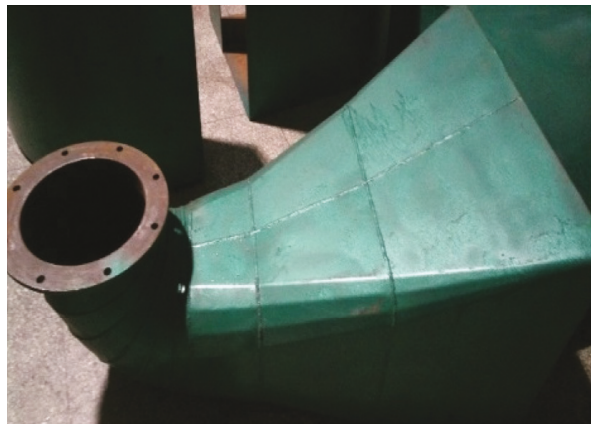

(c)

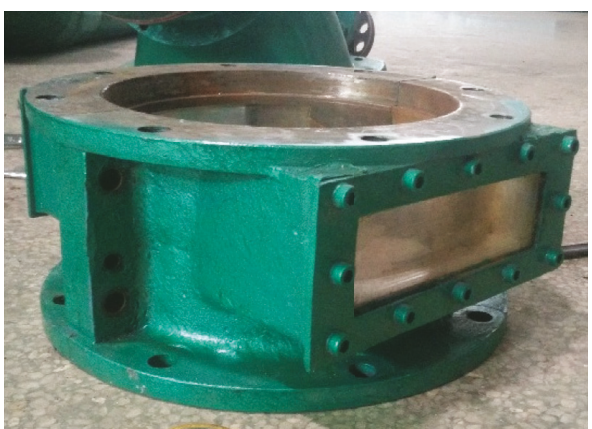

(b)

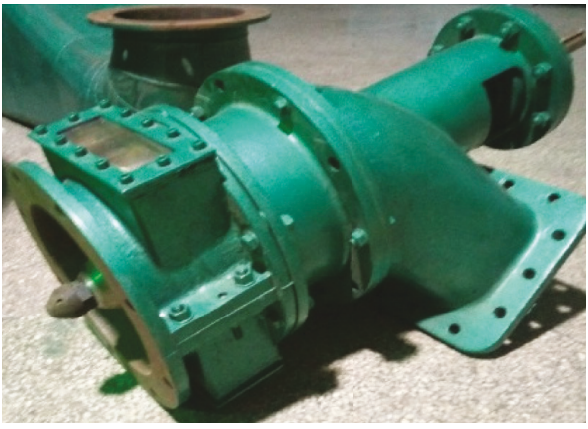

(d)

Figure 10: The device of the whole pump. (a) Blades. (b) Runner chamber. (c) Inlet passage section. (d) Pump device section.

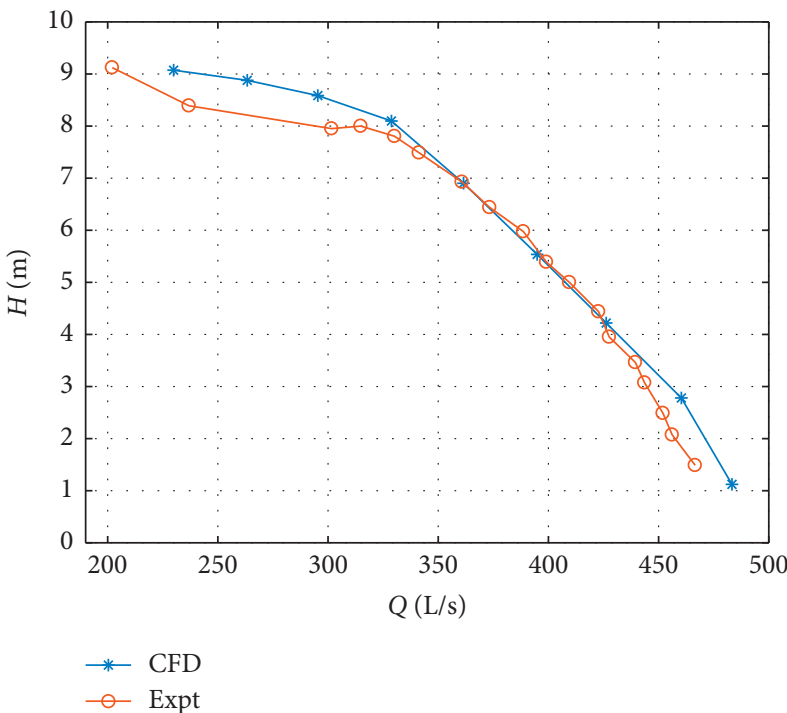

(a)

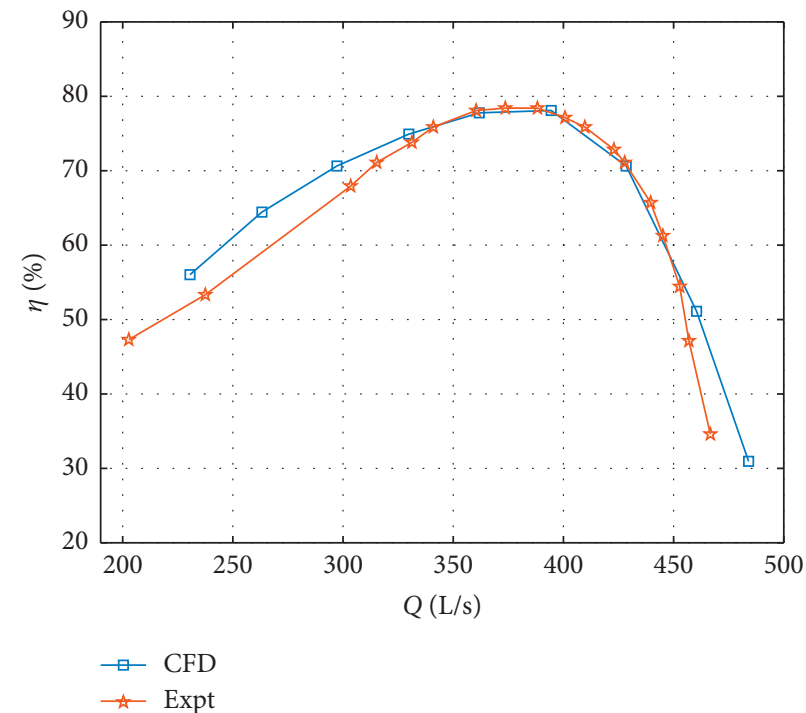

(b)

FIGURE 11: Comparison of model pump test data with numerical simulation data. (a) Head curve. (b) Efficiency curve.

the inlet passage, leading to flow deviation from the ideal condition and thus higher impact losses.

The hydraulic loss ratio of the inlet section is the smallest, whilst the guide vane section's is the largest among the hydraulic loss ratios of each pump section. The change of rotational speed has the greatest influence on the guide vane section, and the corresponding hydraulic loss of $0.5 n$ is 1.0745 times of rated speed's. By contrast, the outlet channel has the smallest hydraulic loss, about 1.043 times the rated speed's. These differences can be justified in part by the fixed rear guide vane used in the hydraulic design of the axial flow pump. Under the design condition, the flow angle of the runner outlet is equal to the inlet placement angle of the guide vane; therefore, the impact loss on the inlet of the guide vane is the smallest. 


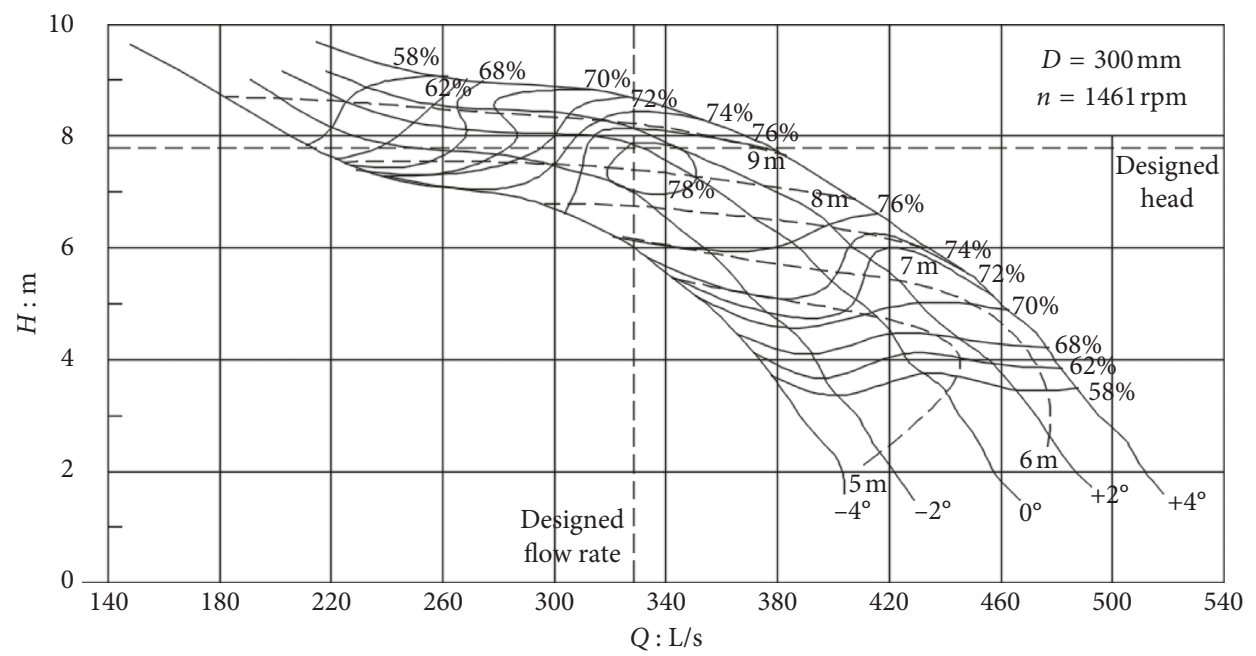

FIGURE 12: Comprehensive characteristic curve.

TABLE 9: Hydraulic loss of the passages.

\begin{tabular}{lccccccc}
\hline \multirow{2}{*}{ Rotational speed (r/min) } & \multicolumn{2}{c}{ Passage of inlet } & \multicolumn{2}{c}{ Passage of guide } & \multicolumn{2}{c}{ Passage of outlet } & Pump efficiency, H (\%) \\
& $K_{\mathrm{p}}(\%)$ & $\delta$ & $K_{\mathrm{p}}(\%)$ & $\delta$ & $K_{\mathrm{p}}(\%)$ & $\delta$ & \\
\hline $1461(n)$ & 1.2257 & 1 & 11.7564 & 1 & 2.3165 & 1 & 93.58 \\
$1315(0.9 n)$ & 1.2351 & 1.0077 & 11.8588 & 1.0087 & 2.3297 & 1.0057 & 93.52 \\
$1169(0.8 n)$ & 1.2478 & 1.0180 & 12.0042 & 1.0211 & 2.3486 & 1.0139 & 93.44 \\
$1023(0.7 n)$ & 1.2527 & 1.0220 & 12.1594 & 1.0343 & 2.3539 & 1.0161 & 93.34 \\
$877(0.6 n)$ & 1.2803 & 1.0445 & 12.3238 & 1.0483 & 2.3887 & 1.0312 & 93.19 \\
$731(0.5 n)$ & 1.3086 & 1.0676 & 12.6327 & 1.0745 & 2.4161 & 1.0430 & 93.09 \\
\hline
\end{tabular}

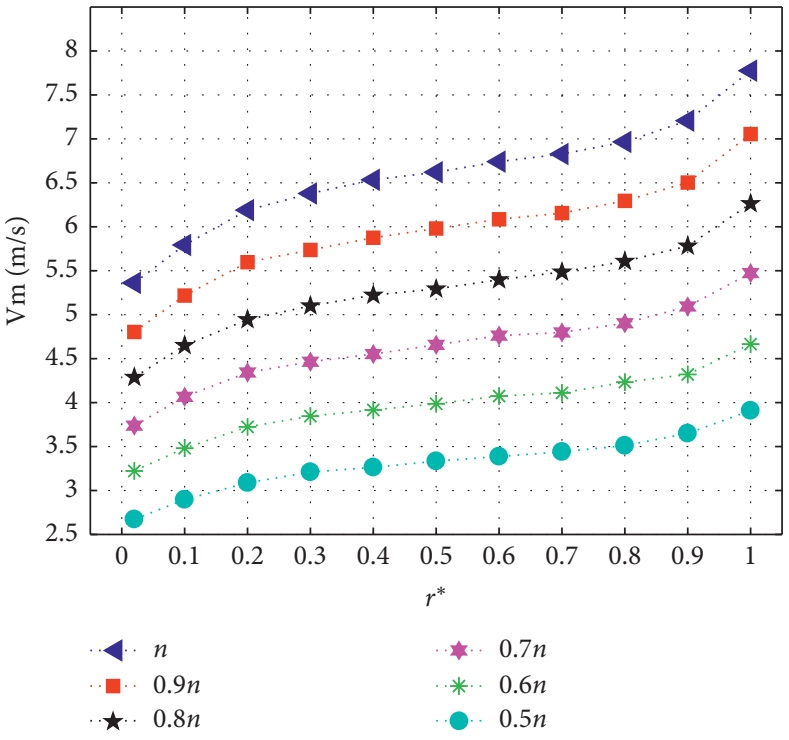

(a)

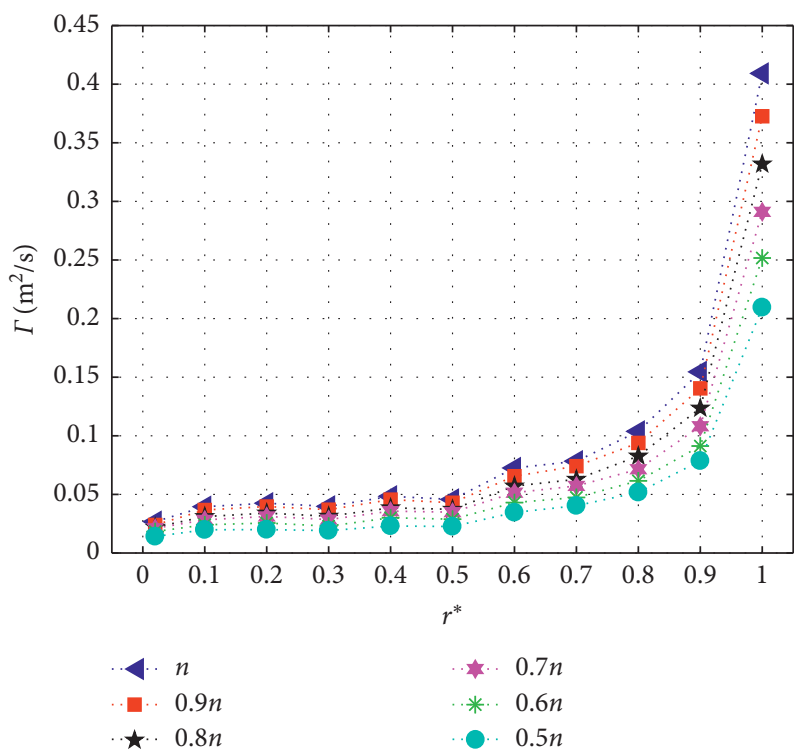

(b)

FIgURE 13: Velocity of the blade inlet section. (a) Axial velocity. (b) Velocity circulation.

4.5.2. Hydraulic Performance of the Blade Inlet and Outlet Section. Figure 13(a) depicts the differences in axial velocity of the blades' inlet section with respect to six rotational speeds. As can be seen, the axial velocity decreases from the tip to the hub and this variation trend with rotational speeds is basically similar. The change of axial velocity with respect 


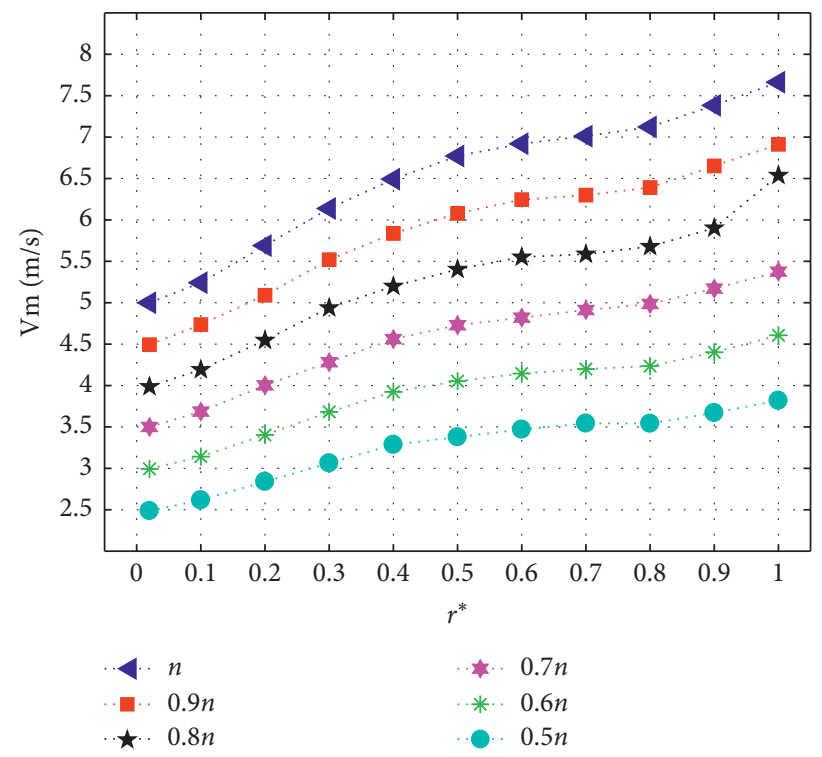

(a)

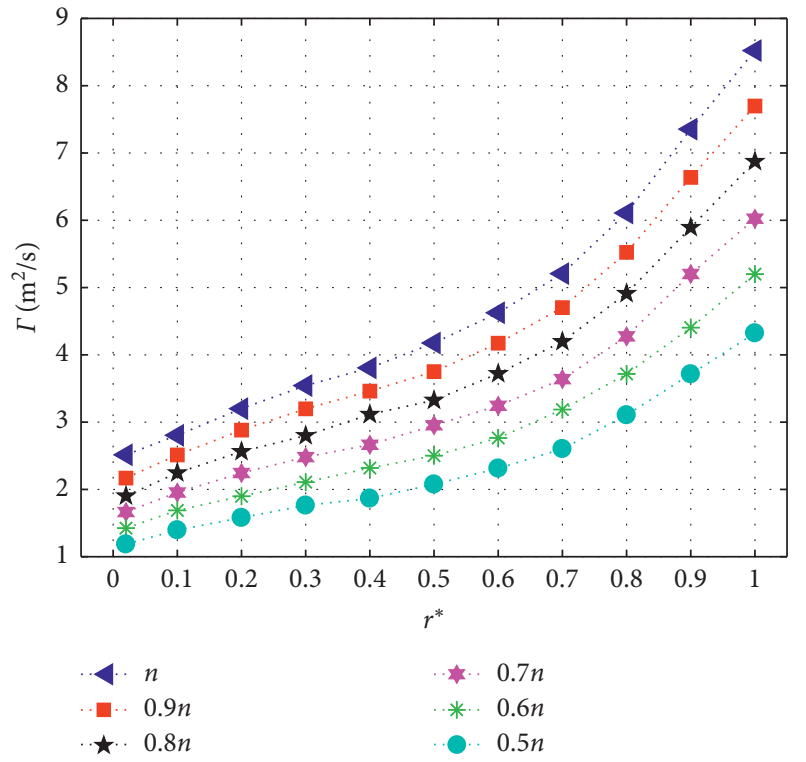

(b)

FIGURE 14: Velocity of the blade outlet section. (a) Axial velocity. (b) Velocity circulation.

to the blade section is largest at the hub and tip, and minimal, at the middle part of the blade. The velocity circulation (Figure 13(b)) increases along the radial direction, according to $r^{*}=\left(r-R_{h}\right) /(R-r)$, where $r$ is the radius of a point at the blade section; $R_{\mathrm{h}}$, the radius of the hub; and $R$, the radius of the blade. The velocity circulation increases smoothly when $r^{*}$ varies from 0.2 to 0.85 , but rises significantly when $r^{*}$ varies from 0.85 to 1 . This correlation can be attributed to the small and large blade setting angle at the hub and at the tip, respectively, resulting in a larger flow circulation through the outer edge of the blade, thus improving the anticavitation performance of the pump.

Figure 14 compares the distributions of axial velocity and velocity circulation at the blade outlet section under the six rotational speed conditions. A monotonous upward trend from hub to tip and low to high speed is observed with both velocities. Compared to the velocity circulation at the blade inlet section, the increase of velocity circulation at the blade outlet section is significantly due to the blade rotation and in direct proportion to the rotational speed.

\section{Conclusion}

The aim of the study was to identify the influence of four interrelated pump factors, with regard to pump's stability and efficiency. To obtain this, a multiobjective optimization design method based on the orthogonal test was adopted. The results of range analysis showed the distance between the guide vane and the blade (factor D) and the hub ratio (factor C) were the least and most influential indexes, respectively. An optimal configuration $\mathrm{A}_{2} \mathrm{~B}_{1} \mathrm{C}_{2} \mathrm{D}_{3}$ was obtained by the comprehensive frequency analysis method, after intuitive and range analysis.

In order to understand how design affected efficiency, a comparison of the flow around the blades and guide vanes was made between the two models. In general, the flow in the two sections was more laminar in the optimized pump than in the original pump, leading to less hydraulic loss and higher efficiency. Furthermore, the high pressure built near the hub of the original pump almost disappears in the optimized design. Under the design point, the optimized axial flow pump was able to meet the requirements of the flow rate and head. In comparison with the nonoptimized model, the new design's pump efficiency increased by $4.26 \%$, whilst the shaft power and pressure coefficient reduced by $1.22 \%$ and $11 \%$, respectively.

The simulations of the pump's head and efficiency were found consistent with experimental results, and the error was less than $5 \%$. The results further prove the validity of the presented methods.

In general, the decrease in rotational speed caused lager hydraulic loss ratio and smaller blade efficiencies. The hydraulic loss ratio of the blade inlet section was the smallest, and that of the guide vane was the largest among the hydraulic loss ratios of each pump section. The change of rotational speed had the greatest influence on the guide vane section. Compared to the velocity circulation at the blade inlet section, the increase of velocity circulation at the blade outlet section was significantly due to the blade rotation, and this was in direct proportion to the rotational speed.

\section{Data Availability}

The parameter data of the optimized pump used to support the findings of this study have not been made available because it is from an important project which involves issues of confidentiality.

\section{Additional Points}

Combined method of CFD and orthogonal test was put forward to optimize pump flow's characteristics. Optimal 
parameters were determined through the comprehensive frequency analysis method. Pressure pulsation was considered as the optimization parameter in the axial flow pump.

\section{Disclosure}

The abstract of the manuscript has been submitted as an oral presentation in 2nd IAHR- Asia Symposium on Hydraulic Machinery and Systems.

\section{Conflicts of Interest}

The authors declare no conflicts of interest.

\section{Authors' Contributions}

Yanhe Xu conceptualized the study; Yuquan Zhang developed the methodology; Aoran Sun was responsible for software; Yuquan Zhang and E. Fernandez-Rodriguez validated the data; Yuan Zheng performed the formal analysis; Yanhe $\mathrm{Xu}$ and Yuquan Zhang were involved in investigation; Yanhe $\mathrm{Xu}$ was responsible for resources; Yuquan Zhang and E. Fernandez-Rodriguez prepared the original draft; Chunxia Yang and Jue Wang reviewed and edited the manuscript; Yuan Zheng supervised the study; Yuquan Zhang and Yanhe $\mathrm{Xu}$ were responsible for funding acquisition.

\section{Acknowledgments}

The research was supported by the following funding programs: National Natural Science Foundation of China (51809083), Natural Science Foundation of Jiangsu Province (BK20180504), Fundamental Research Funds for the Central Universities (no. 2019B15114), China Postdoctoral Science Foundation (2019M651678), and Water Conservancy Science \& Technology Project of Jiangsu Province (2018026).

\section{References}

[1] C. Wang, W. Shi, X. Wang et al., "Optimal design of multistage centrifugal pump based on the combined energy loss model and computational fluid dynamics," Applied Energy, vol. 187, pp. 10-26, 2017.

[2] R. H. Almeida, J. R. Ledesma, I. B. Carrêlo, L. Narvarte, G. Ferrara, and L. Antipodi, "A new pump selection method for large-power PV irrigation systems at a variable frequency," Energy Conversion and Management, vol. 174, pp. 874-885, 2018.

[3] P. Olszewski and J. Arafeh, "Parametric analysis of pumping station with parallel-configured centrifugal pumps towards self-learning applications," Applied Energy, vol. 231, pp. 1146-1158, 2018.

[4] M. Liu, R. Ooka, W. Choi, and S. Ikeda, "Experimental and numerical investigation of energy saving potential of centralized and decentralized pumping systems," Applied Energy, vol. 251, Article ID 113359, 2019.

[5] S. Lei, D. S. Zhang, R. J. Zhao, W. Shi, and Y. Jin, "Effect of blade tip geometry on tip leakage vortex dynamics and cavitation pattern in axial-flow pump," Science China Technological Sciences, vol. 60, no. 10, pp. 1480-1493, 2017.
[6] Z. Qian, F. Wang, Z. Guo, and J. Lu, "Performance evaluation of an axial-flow pump with adjustable guide vanes in turbine mode," Renewable Energy, vol. 99, pp. 1146-1152, 2016.

[7] D. Zhang, W. Shi, B. P. M. van Esch, L. Shi, and M. Dubuisson, "Numerical and experimental investigation of tip leakage vortex trajectory and dynamics in an axial flow pump," Computers \& Fluids, vol. 112, no. 1, pp. 61-71, 2015.

[8] M. Binama, W.-T. Su, W.-H. Cai et al., "Blade trailing edge position influencing pump as turbine (PAT) pressure field under part-load conditions," Renewable Energy, vol. 136, pp. 33-47, 2019.

[9] Y. Long, D. Wang, J. Yin, and Y. Hu, "Experimental investigation on the unsteady pressure pulsation of reactor coolant pumps with non-uniform inflow," Annals of Nuclear Energy, vol. 110, pp. 501-510, 2017.

[10] S. I. Ngo, Y.-I. Lim, W. Kim, D. J. Seo, and W. L. Yoon, "Computational fluid dynamics and experimental validation of a compact steam methane reformer for hydrogen production from natural gas," Applied Energy, vol. 236, pp. 340-353, 2019.

[11] S.-Q. Wang, K. Sun, G. Xu, Y.-T. Liu, and X. Bai, "Hydrodynamic analysis of horizontal-axis tidal current turbine with rolling and surging coupled motions," Renewable Energy, vol. 102, pp. 87-97, 2017.

[12] X.-F. Lin, J.-S. Zhang, Y.-Q. Zhang, J. Zhang, and S. Liu, "Comparison of actuator line method and full rotor geometry simulations of the wake field of a tidal stream turbine," Water, vol. 11, no. 3, p. 560, 2019.

[13] T. Korakianitis, I. A. Hamakhan, M. A. Rezaienia, A. P. S. Wheeler, E. J. Avital, and J. J. R. Williams, "Design of high-efficiency turbomachinery blades for energy conversion devices with the three-dimensional prescribed surface curvature distribution blade design (CIRCLE) method," Applied Energy, vol. 89, no. 1, pp. 215-227, 2012.

[14] Y. Zhang, J. Zhang, Y. Zheng, C. Yang, W. Zang, and E. Fernandez-Rodriguez, "Experimental analysis and evaluation of the numerical prediction of wake characteristics of tidal stream turbine," Energies, vol. 10, no. 12, p. 2057, 2017.

[15] T. Ouchbel, S. Zouggar, M. L. Elhafyani et al., "Power maximization of an asynchronous wind turbine with a variable speed feeding a centrifugal pump," Energy Conversion and Management, vol. 78, no. 2, pp. 976-984, 2014.

[16] Y. Zhang, Y. Zheng, E. Fernandez-Rodriguez et al., "Optimization design of submerged propeller in oxidation ditch by computational fluid dynamics and comparison with experiments," Water Science and Technology, vol. 74, no. 3, pp. 681-690, 2016.

[17] M. H. Shojaeefard, M. Tahani, M. B. Ehghaghi, M. A. Fallahian, and M. Beglari, "Numerical study of the effects of some geometric characteristics of a centrifugal pump impeller that pumps a viscous fluid," Computers \& Fluids, vol. 60 , no. 4, pp. 61-70, 2012.

[18] M. Asuaje, F. Bakir, S. Kouidri, and R. Rey, "Inverse design method for centrifugal impellers and comparison with numerical simulation tools," International Journal of Computational Fluid Dynamics, vol. 18, no. 2, pp. 101-110, 2004.

[19] R. Spence and J. Amaral-Teixeira, "A CFD parametric study of geometrical variations on the pressure pulsations and performance characteristics of a centrifugal pump," Computers \& Fluids, vol. 38, no. 6, pp. 1243-1257, 2009.

[20] A. Fleder and M. Böhle, "A systematical study of the influence of blade number on the performance of a side channel pump," Journal of Fluids Engineering, vol. 141, no. 11, p. 111109, 2019. 
[21] F. Yang, H. Zhao, and C. Liu, "Improvement of the efficiency of the axial-flow pump at part loads due to installing outlet guide vanes mechanism," Mathematical Problems in Engineering, vol. 2016, Article ID 6375314, 10 pages, 2016.

[22] H. Hou, Y. Zhang, X. Zhou, Z. Zuo, and H. Chen, "Optimal hydraulic design of an ultra-low specific speed centrifugal pump based on the local entropy production theory," Proceedings of the Institution of Mechanical Engineers, Part A: Journal of Power and Energy, vol. 233, no. 6, pp. 715-726, 2019.

[23] H.-C. Park, S. Kim, J.-Y. Yoon, and Y.-S. Choi, “A numerical study on the performance improvement of guide vanes in an axial-flow pump," Journal of Fluid Machinery, vol. 15, no. 6, pp. 58-63, 2012.

[24] Y. Liu, L. Tan, M. Liu, Y. Hao, and Y. Xu, "Influence of prewhirl angle and axial distance on energy performance and pressure fluctuation for a centrifugal pump with inlet guide vanes," Energies, vol. 10, no. 5, p. 695, 2017.

[25] D. Bonaiuti, M. Zangeneh, R. Aartojarvi, and J. Eriksson, "Parametric design of a waterjet pump by means of inverse design, CFD calculations and experimental analyses," Journal of Fluids Engineering, vol. 132, no. 3, 2010.

[26] L. Yun, Z. Rongsheng, W. Dezhong, Y. Junlian, and L. Tianbin, "Numerical and experimental investigation on the diffuser optimization of a reactor coolant pump with orthogonal test approach," Journal of Mechanical Science and Technology, vol. 30, no. 11, pp. 4941-4948, 2016.

[27] J. Tang, G. Gong, H. Su, F. Wu, and C. Herman, "Performance evaluation of a novel method of frost prevention and retardation for air source heat pumps using the orthogonal experiment design method," Applied Energy, vol. 169, pp. 696-708, 2016.

[28] Y. W. Liu, R. J. Wu, P. Yang, T. G. Wang, H. H. Liu, and L. H. Wang, "Parameter study of the injection configuration in a zero boil-off hydrogen storage tank using orthogonal test design," Applied Thermal Engineering, vol. 109, pp. 283-294, 2016.

[29] L. Tang, S. Yuan, and Y. Tang, "Performance improvement of a micro impulse water turbine based on orthogonal array," Mathematical Problems in Engineering, vol. 2017, Article ID 5867101, 15 pages, 2017.

[30] D. M. Byrne, "The Taguchi approach to parameter design," ASQ's Annual Quality Congress Proceedings, vol. 40, pp. 168-177, 1986.

[31] Y. Xu, L. Tan, S. Cao, and W. Qu, "Multiparameter and multiobjective optimization design of centrifugal pump based on orthogonal method," Proceedings of the Institution of Mechanical Engineers, Part C: Journal of Mechanical Engineering Science, vol. 231, no. 14, pp. 2569-2579, 2017.

[32] L. Zhou, W. Shi, and S. Wu, "Performance optimization in a centrifugal pump impeller by orthogonal experiment and numerical simulation," Advances in Mechanical Engineering, vol. 5, pp. 31-41, 2013.

[33] C. Wang, W. Shi, L. Zhou, and W. Lu, "Effect analysis of geometric parameters on stainless steel stamping multistage pump by experimental test and numerical calculation," Advances in Mechanical Engineering, vol. 5, pp. 1-8, 2013.

[34] L. Shi, F. Tang, R. Xie, and W. Zhang, "Numerical and experimental investigation of tank-type axial-flow pump device," Advances in Mechanical Engineering, vol. 9, no. 3, Article ID 168781401769568, 2017.

[35] J. Zhang, H. Zhu, C. Yang, Y. Li, and H. Wei, "Multi-objective shape optimization of helico-axial multiphase pump impeller based on NSGA-II and ANN," Energy Conversion and Management, vol. 52, no. 1, pp. 538-546, 2011.

[36] D. Ye, X. Lai, H. Li, and X. Zhang, "Optimal design and experiment of exhaust vent on impeller of medium consistency pulp pump," Advances in Mechanical Engineering, vol. 10, no. 3, Article ID 1687814018765557, 2018.

[37] Y. Wang, X. Huo, and H. Ji, "Multiobjective optimization design and experimental study of desulfurization dust removal centrifugal pump based on immune particle swarm algorithm," Advances in Materials Science and Engineering, vol. 2018, Article ID 6218178, 10 pages, 2018.

[38] W. Tao, L. Yimo, and L. Lin, "A novel 3D simulation model for investigating liquid desiccant dehumidification performance based on CFD technology," Applied Energy, vol. 240, pp. 486-498, 2019.

[39] F. R. Menter, "Review of the shear-stress transport turbulence model experience from an industrial perspective," International Journal of Computational Fluid Dynamics, vol. 23, no. 4, pp. 305-316, 2009.

[40] J. Zheng, C. Zhang, Z. Demirbilek, and L. Lin, "Numerical study of sandbar migration under wave-undertow interaction," Journal of Waterway, Port, Coastal, and Ocean Engineering, vol. 140, no. 2, pp. 146-159, 2014.

[41] J. Zhang, H.-H. Zhang, Y.-L. He, and W.-Q. Tao, "A comprehensive review on advances and applications of industrial heat pumps based on the practices in China," Applied Energy, vol. 178, pp. 800-825, 2016. 


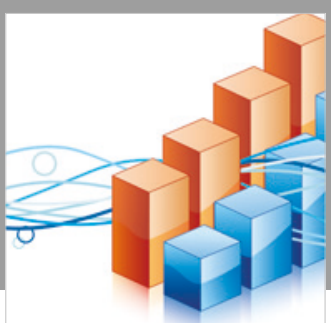

Advances in

Operations Research

\section{-n-m}
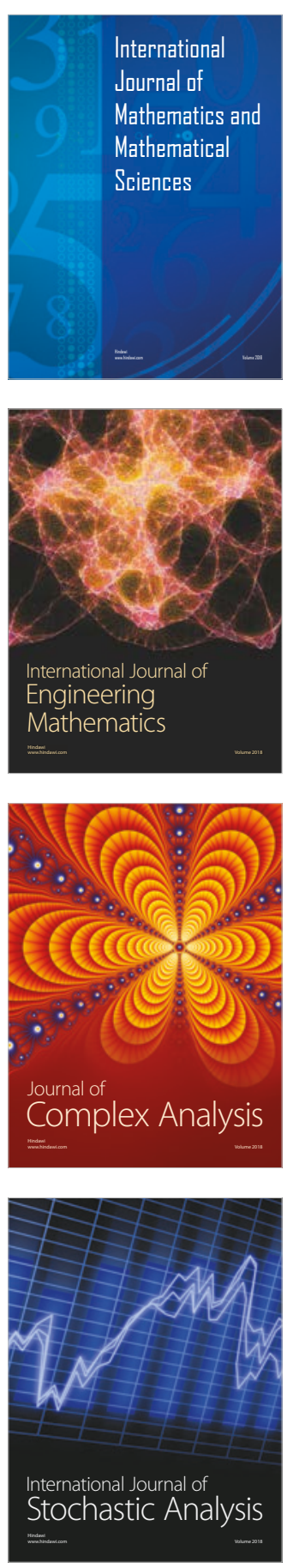
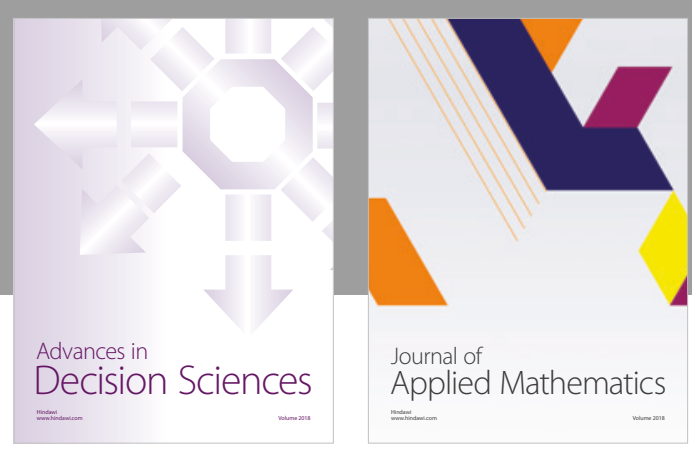

Journal of

Applied Mathematics
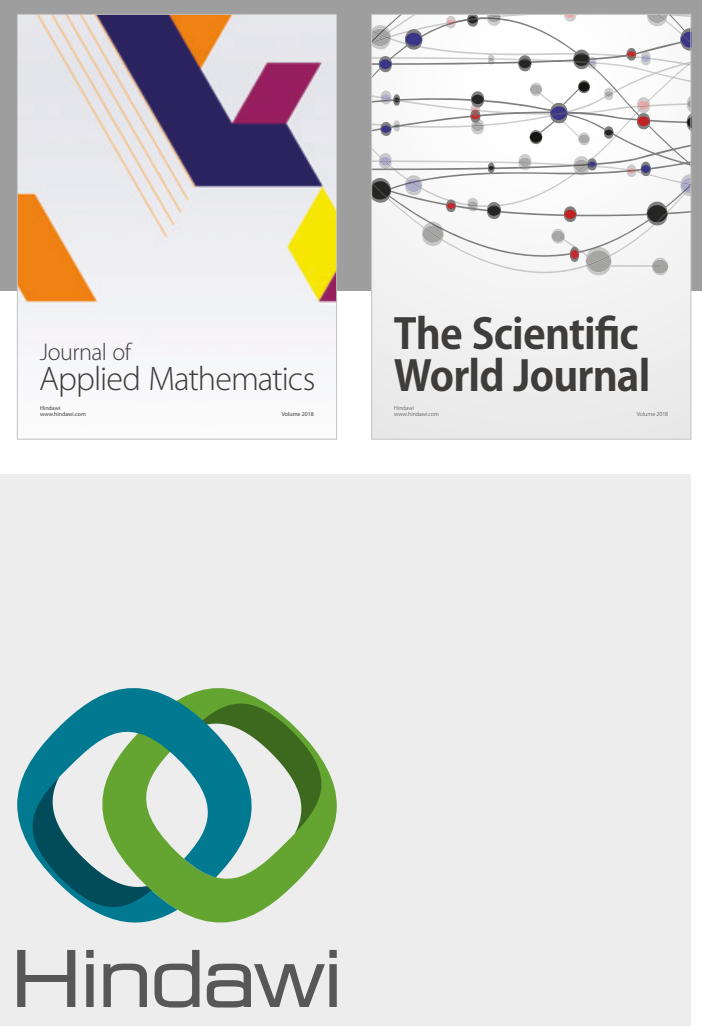

Submit your manuscripts at

www.hindawi.com

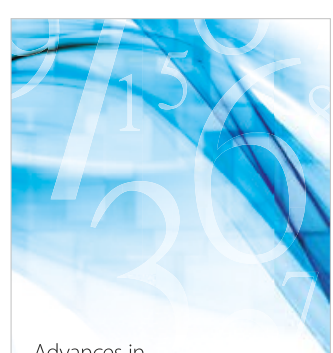

Advances in
Numerical Analysis
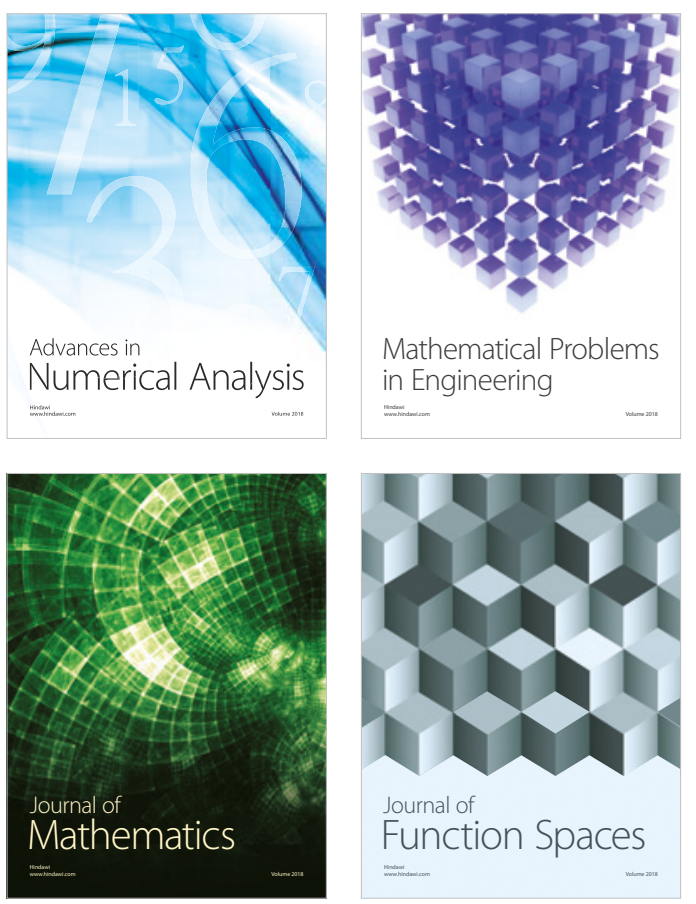

Mathematical Problems in Engineering

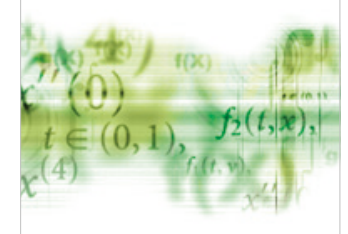

International Journal of

Differential Equations

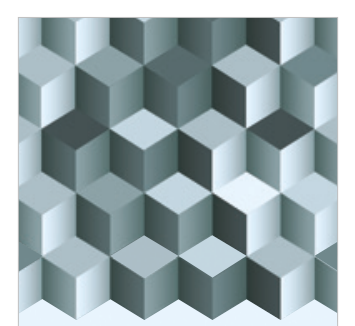

Journal of

Function Spaces
The Scientific

World Journal

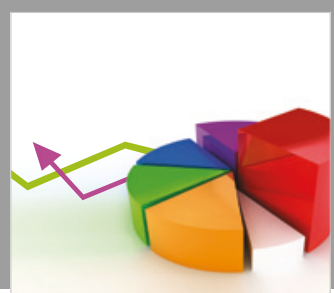

Journal of

Probability and Statistics
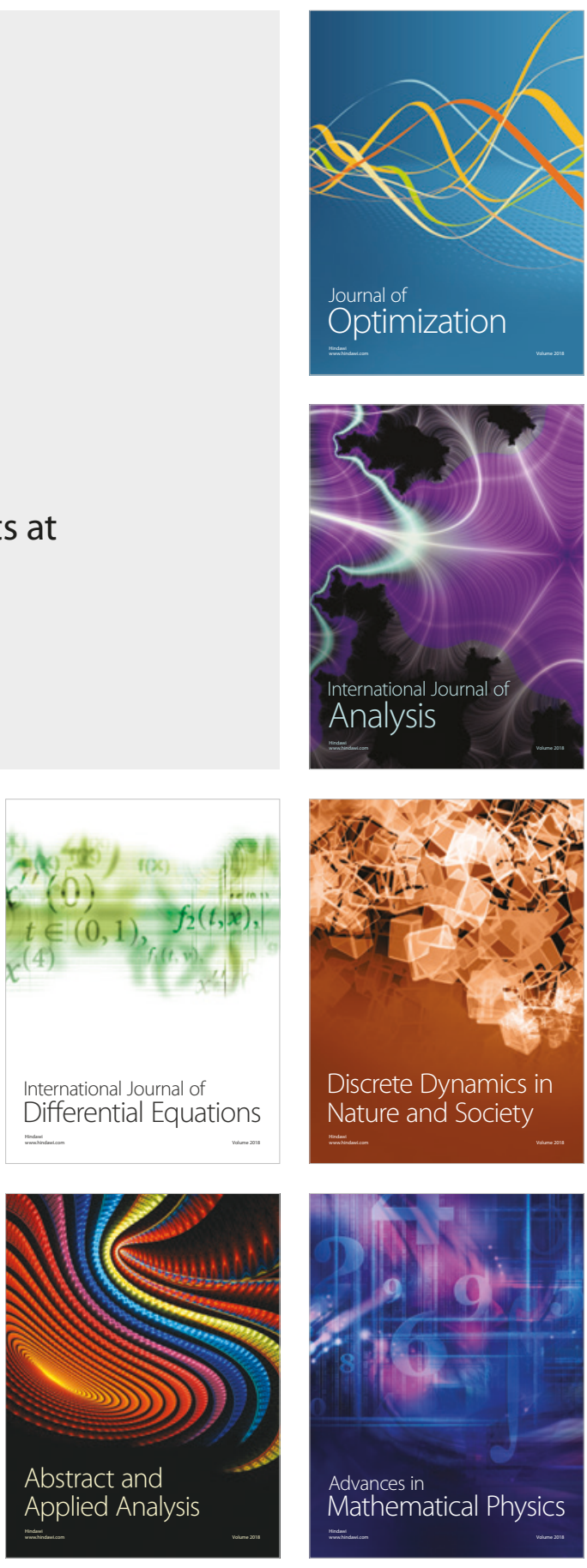